NBER WORKING PAPER SERIES

\title{
CLIMATE ECONOMETRICS
}

Solomon M. Hsiang

Working Paper 22181

http://www.nber.org/papers/w22181

\author{
NATIONAL BUREAU OF ECONOMIC RESEARCH \\ 1050 Massachusetts Avenue \\ Cambridge, MA 02138 \\ April 2016
}

I thank David Anthoff, Jesse Anttila-Hughes, Max Auffhammer, Alan Barrecca, Marshall Burke, Tamma Carleton, Olivier Deschenes, Tatyana Deryugina, Ram Fishman, Michael Greenstone, Michael Hanemann, Wu-Teh Hsiang, Bob Kopp, David Lobell, Gordon McCord, Kyle Meng, Billy Pizer, James Rising, Michael Roberts, Wolfram Schlenker, Christian Traeger, and seminar participants at Berkeley and Harvard for discussions and suggestions. I thank to Wolfram Schlenker for generously sharing data. The views expressed herein are those of the authors and do not necessarily reflect the views of the National Bureau of Economic Research.

NBER working papers are circulated for discussion and comment purposes. They have not been peer-reviewed or been subject to the review by the NBER Board of Directors that accompanies official NBER publications.

(C) 2016 by Solomon M. Hsiang. All rights reserved. Short sections of text, not to exceed two paragraphs, may be quoted without explicit permission provided that full credit, including (C) notice, is given to the source. 
Climate Econometrics

Solomon M. Hsiang

NBER Working Paper No. 22181

April 2016

JEL No. C33,H84,I1,O13,Q54

\begin{abstract}
$\underline{\text { ABSTRACT }}$
Identifying the effect of climate on societies is central to understanding historical economic development, designing modern policies that react to climatic events, and managing future global climate change. Here, I review, synthesize, and interpret recent advances in methods used to measure effects of climate on social and economic outcomes. Because weather variation plays a large role in recent progress, I formalize the relationship between climate and weather from an econometric perspective and discuss their use as identifying variation, highlighting tradeoffs between key assumptions in different research designs and deriving conditions when weather variation exactly identifies the effects of climate. I then describe advances in recent years, such as parameterization of climate variables from a social perspective, nonlinear models with spatial and temporal displacement, characterizing uncertainty, measurement of adaptation, cross-study comparison, and use of empirical estimates to project the impact of future climate change. I conclude by discussing remaining methodological challenges.
\end{abstract}

Solomon M. Hsiang

Goldman School of Public Policy

University of California, Berkeley

2607 Hearst Avenue

Berkeley, CA 94720-7320

and NBER

shsiang@berkeley.edu 


\section{INTRODUCTION}

How does the climate affect society and the economy? This question has challenged thinkers for centuries - the answer promises insight into why economies developed differently historically, how modern society can best respond to current climatic events, and how future climate changes may impact humanity. In recent years, numerous econometric analyses have emerged addressing this question by studying the effects of specific climatic conditions on different social and economic outcomes. The recency of this research activity is explained primarily by methodological advances that, combined with increasing access to computing power and climate data, catalyzed progress.

The goal of this article is to collect and synthesize these advances. In particular, I try to highlight core innovations and to explain linkages between different methods. I also attempt to tackle an issue that has proved particularly thorny: a debate as to whether regressions on "weather" variables provide meaningful insight into the effects of climate. By formalizing this question, I am able to derive conditions under which the use of weather variables in regressions is justified and, perhaps surprisingly, dominates traditionally preferred methods. In the latter portion of this article, I discuss how these new econometric results are being used to understand other scientific or policy questions, such as the optimal design of climate change policy. Throughout, I draw attention to methodological challenges that remain unsolved.

This article focuses on methodology, so I will not describe data or results that are not examples of methodological innovations. I encourage readers to consult Auffhammer et al. (2013) for a discussion of climate data generally, and other review articles surveying findings from this rapidly growing field: for health impacts see Deschênes (2014), for agricultural impacts see Auffhammer \& Schlenker (2014), for energy impacts see Auffhammer \& Mansur (2014), for conflict impacts see Burke et al. (2015b), for climatic disaster impacts broadly speaking Kousky (2014) and for tropical cyclones specifically see Camargo \& Hsiang (2016), for labor impacts see Heal \& Park (2015), and for a general summary of findings from across the literature see Dell et al. (2014) and Carleton \& Hsiang (2016).

\subsection{Defining climate}

Here I develop a formal definition for the climate that is flexible, general, and encompasses usages throughout the literature.

For any position in space $i$, there exists a vector of random variables at each moment in time $t$ characterizing the conditions of the atmosphere and ocean that are relevant to economic conditions at $i$. Heuristically, one could imagine this random vector as

$$
\mathbf{v}_{i t}=\left[\text { temperature }_{i t}, \text { precipitation }_{i t}, \text { humidity }_{i t}, \ldots\right]
$$

For an interval in time $\tau=[\underline{t}, \bar{t})$ at $i$, there exists a joint probability distribution $\psi\left(\mathbf{C}_{i \tau}\right)$ from which we imagine $\mathbf{v}_{i t}$ is drawn:

$$
\mathbf{v}_{i t} \sim \psi\left(\mathbf{C}_{i \tau}\right) \quad \forall t \in \tau
$$

$\mathbf{C}_{i \tau}$ is a vector of $K$ relevant parameters - ideally sufficient statistics - indexed by $k$, that characterize distributions in the $\psi($.$) family of distributions, such as location and shape parameters. Define \mathbf{C}_{i \tau}$ to be the climate at $i$ during $\tau$, since it characterizes the distribution of possible realized states $\mathbf{v}_{i t}$.

For each period $\tau$, there is an empirical distribution $\psi\left(\mathbf{c}_{i \tau}\right)$ that characterizes the distribution of states $\mathbf{v}_{i, t \in \tau}$ that are actually realized. In many contexts, some of the $K$ parameters in $\mathbf{c}_{i \tau}$ have analogs to fitted values for a model where the distribution is constrained to the $\psi($.$) family, but such an analogy is imperfect because \mathbf{c}_{i \tau}$ are actual measurements, not estimates ${ }^{1}$. Note that $\mathbf{c}_{i \tau}$ and $\mathbf{C}_{i \tau}$ are vectors of the same length with analogous

\footnotetext{
${ }^{1}$ It is possible that some researchers may attempt to construct empirical estimates of $\hat{\mathbf{C}}_{i \tau}$ using data that resemble or are identical to measurements $\mathbf{c}_{i \tau}$, but this need not always be the case. For example, an estimate for the population mean of daily temperatures
} 
elements, but they are not the same. $\mathbf{C}_{i \tau}$ characterizes the expected distribution of $\mathbf{v}_{i t}$ while $\mathbf{c}_{i \tau}$ characterizes the realized distribution of $\mathbf{v}_{i, t \in \tau}$. Thus, we define $\mathbf{c}_{i \tau}$ to be a description of the weather during $\tau$.

Examples help clarify how these definitions of climate and weather differ. Consider that weather measures $\mathbf{c}_{i \tau}$ might contain the sample mean and sample standard deviation of daily rainfall during a month, whereas the corresponding $\mathbf{C}_{i \tau}$ would contain the true population mean and true population standard deviation of rainfall that could occur during that period. In another example, $\mathbf{c}_{i \tau}$ could contain the maximum sustained wind gust speed actually experienced during a 24 hour interval while $\mathbf{C}_{i \tau}$ contains the maximum of the true theoretical gust distribution for that day. Finally, $\mathbf{c}_{i \tau}$ could contain the count of realized days with average temperatures below freezing or above $30^{\circ} \mathrm{C}$ in a year, whereas $\mathbf{C}_{i \tau}$ might then contain the expected number of days in these categories.

For notational simplicity, define $\mathbf{c}(\mathbf{C})$ as a realization of weather characteristics $\mathbf{c}$ conditional on climate characteristics $\mathbf{C}$.

Two questions immediately emerge for an applied econometrician. First, how should the joint distribution $\psi(\mathbf{C})$ for the high dimensional vector $\mathbf{v}$ be summarized? Are we concerned only with average values and variances, or some other summary statistics, such as time beyond a critical value (e.g. extreme heat days) or events that involve multiple dimensions of $\mathbf{v}$ (e.g. wind and rain simultaneously)? Unfortunately, at present there is no exhaustive list of summary statistics or dimensions of $\mathbf{v}$ that fully describes all socially and economically relevant parameters. In practice, different researchers have explored whether and how different summary measures c matter by examining one or a few at a time, for example examining average temperatures when controlling for average rainfall, but these should be understood as rough characterizations of a more highly structured multi-dimensional distribution. As current research progresses, the set of known relevant summary parameters generally tends to grow.

Second, how long of a time interval $\tau$ should be considered? Historically, climate was sometimes defined as an average over 30 years (Pachauri et al., 2014), but this definition is fairly arbitrary. In reality, there exists a well-defined expected distribution of states that might occur even for very short periods of time. For example, at every location there is an expected distribution of temperatures that might occur for each five-minute interval on each day of the year. Furthermore, this distribution might change between consecutive years, for example, due to the El Niño-Southern Oscillation (ENSO). This suggests that climate need not have a fundamental time-scale and econometricians may, in principle, study periods of varying lengths of time.

\subsection{Influence of climate through events and information}

The climate affects social outcomes in two ways. First, the climate during $\tau$ influences what realizations of weather c actually occur during that interval, which in turn affects a population directly (e.g. a rainy climate generates rain, causing people to get wet) - call this the "direct effect" of climate. Second, individuals' beliefs over the structure of $\mathbf{C}$ may affect their decisions and resulting outcomes, regardless of what $\mathbf{c}$ is realized (e.g. if people believe their climate is rainy, some will buy umbrellas) - refer to this as the "belief effect." Denote all actions resulting from beliefs as the vector $\mathbf{b}$ of length $N$, indexed by $n$. We can then write that an outcome is affected by the climate because the climate affects what weather is realized and what actions individuals take based on their beliefs about the climate

$$
Y(\mathbf{C})=Y(\mathbf{c}(\mathbf{C}), \mathbf{b}(\mathbf{C})) .
$$

during a year, a climate parameter, happens to equal the sample mean of daily temperatures, a weather parameter. But weather parameters need not always have the same form as estimators for climate parameters and climate parameters, describing an abstract population distribution that is never actually observed, need not depend on weather. Weather parameters should always be interpreted as measurements associated with individual observations. In principle, climate parameters could be formulated in the absence of real world measurements, for example based on a theoretical or numerical model of the climate. 
Therefore the total marginal effect of the climate on outcome $Y$ is characterized by the $K$-element vector of derivatives

$$
\begin{aligned}
\frac{\mathrm{d} Y(\mathbf{C})}{\mathrm{d} \mathbf{C}} & =\nabla_{\mathbf{c}} Y(\mathbf{C}) \cdot \frac{\mathrm{d} \mathbf{c}}{\mathrm{d} \mathbf{C}}+\nabla_{\mathbf{b}} Y(\mathbf{C}) \cdot \frac{\mathrm{d} \mathbf{b}}{\mathrm{d} \mathbf{C}} \\
& =\underbrace{\sum_{k=1}^{K} \frac{\partial Y(\mathbf{C})}{\partial \mathbf{c}_{k}} \frac{\mathrm{d} \mathbf{c}_{k}}{\mathrm{~d} \mathbf{C}}}_{\text {"direct effects" }}+\underbrace{\sum_{n=1}^{N} \frac{\partial Y(\mathbf{C})}{\partial \mathbf{b}_{n}} \frac{\mathrm{d} \mathbf{b}_{n}}{\mathrm{~d} \mathbf{C}}}_{\text {"belief effects" }}
\end{aligned}
$$

where $\nabla_{\mathbf{c}}$ and $\nabla_{\mathbf{b}}$ are defined as gradients in the subspaces of $\mathbf{c}$ and $\mathbf{b}$, respectively. ${ }^{2}$ Observe that $\frac{\mathrm{d} \mathbf{c}}{\mathrm{d} \mathbf{C}}$ and $\frac{\mathrm{d} \mathbf{b}}{\mathrm{d} \mathbf{C}}$ are $K \times K$ and $N \times K$ Jacobians. ${ }^{3}$

Note that all partial derivatives are evaluated "locally" at the current climate C. This local-ness is important, since beliefs about the climate may alter $\frac{\partial Y}{\partial \mathbf{c}_{k}}$ if actions individuals take based on these beliefs alter the direct effect of weather realizations $\mathbf{c}$ when they occur (e.g. individuals who buy umbrellas because they believe they are in a rainy climate get less wet when it rains). Such interactions between beliefs and direct impacts $\left(\frac{\partial^{2} Y}{\partial \mathbf{b}_{n} \partial \mathbf{c}_{k}}\right)$ and belief effects themselves are together often referred to as "adaptations" in the literature.

Researchers are generally interested in both pathways of influence, although credibly identifying belief effects has proven challenging because beliefs are difficult to observe and they tend to be correlated with many other factors.

\section{THE EMPIRICAL PROBLEM}

We are interested in identifying the effect of the climate on a population or economy, holding all other factors fixed. Denoting the vector of observable non-climatic factors $\mathbf{x}$ that affect outcome $Y$, we can express the average treatment effect $\beta$ for a change in climate $\Delta \mathbf{C}_{i \tau}$ as

$$
\beta=\mathrm{E}\left[Y_{i \tau} \mid \mathbf{C}_{i \tau}+\Delta \mathbf{C}_{i \tau}, \mathbf{x}_{i \tau}\right]-\mathrm{E}\left[Y_{i \tau} \mid \mathbf{C}_{i \tau}, \mathbf{x}_{i \tau}\right]
$$

Inference is challenging because $\beta$ can never be observed directly, since the single population $i$ can never be exposed to both counterfactuals $\mathbf{C}$ and $\mathbf{C}+\Delta \mathbf{C}$ for the exact same interval of time $\tau$. This is the Fundamental Problem of Causal Inference (Holland, 1986).

In an ideal experiment aimed at recovering $\beta$, we would locate two sample populations $(i$ and $j$ ) that are identical in every way and experimentally manipulate the climate of $i$ to be $\mathbf{C}$ and the climate of $j$ to be $\mathbf{C}+\Delta \mathbf{C}$. We would then observe how these two treatments affect the outcome $Y$. If they are identical, it must be true that

$$
\mathrm{E}\left[Y_{i \tau} \mid \mathbf{C}, \mathbf{x}_{i \tau}\right]=\mathrm{E}\left[Y_{j \tau} \mid \mathbf{C}, \mathbf{x}_{j \tau}\right]
$$

the unit homogeneity assumption. Note that the right-hand side term is not observed. We could then use observations from our experiment to construct the unbiased estimator

$$
\hat{\beta}=\mathrm{E}\left[Y_{j \tau} \mid \mathbf{C}+\Delta \mathbf{C}, \mathbf{x}_{j \tau}\right]-\mathrm{E}\left[Y_{i \tau} \mid \mathbf{C}, \mathbf{x}_{i \tau}\right]=\underbrace{\mathrm{E}\left[Y_{i \tau} \mid \mathbf{C}+\Delta \mathbf{C}, \mathbf{x}_{i \tau}\right]}_{\text {never observed }}-\mathrm{E}\left[Y_{i \tau} \mid \mathbf{C}, \mathbf{x}_{i \tau}\right]=\beta .
$$

\footnotetext{
${ }^{2}$ Define $\nabla_{\mathbf{c}} Y=\left[\frac{\partial Y}{\partial \mathbf{c}_{1}}, \cdots, \frac{\partial Y}{\partial \mathbf{c}_{K}}\right]$ and $\nabla_{\mathbf{b}} Y=\left[\frac{\partial Y}{\partial \mathbf{b}_{1}}, \cdots, \frac{\partial Y}{\partial \mathbf{b}_{N}}\right]$ which can be concatenated to form the complete gradient vector $\nabla Y=\left[\nabla_{\mathbf{c}} Y, \nabla_{\mathbf{b}} Y\right]$

3 The Jacobian matrices are $\frac{\mathrm{d} \mathbf{c}}{\mathrm{d} \mathbf{C}}=\left[\begin{array}{ccc}\frac{\partial \mathbf{c}_{1}}{\partial \mathbf{C}_{1}} & \cdots & \frac{\partial \mathbf{c}_{1}}{\partial \mathbf{C}_{K}} \\ \vdots & \ddots & \vdots \\ \frac{\partial \mathbf{c}_{K}}{\partial \mathbf{C}_{1}} & \cdots & \frac{\partial \mathbf{c}_{K}}{\partial \mathbf{C}_{K}}\end{array}\right]$ and $\frac{\mathrm{d} \mathbf{b}}{\mathrm{d} \mathbf{C}}=\left[\begin{array}{ccc}\frac{\partial \mathbf{b}_{1}}{\partial \mathbf{C}_{1}} & \cdots & \frac{\partial \mathbf{b}_{1}}{\partial \mathbf{C}_{K}} \\ \vdots & \ddots & \vdots \\ \frac{\partial \mathbf{b}_{N}}{\partial \mathbf{C}_{1}} & \cdots & \frac{\partial \mathbf{b}_{N}}{\partial \mathbf{C}_{K}}\end{array}\right]$.
} 
Unfortunately, such an experiment is usually impossible for most large-scale settings of interest, although some laboratory experiments have applied a randomized version of this approach in psychology (Mackworth, 1946), ergonomics (Seppanen et al., 2006), sports medicine (Nybo \& Secher, 2004), and military research (Hocking et al., 2001). In these settings, where $\Delta \mathbf{C}$ can be randomly assigned and experimentally manipulated (e.g. warming a room), application of Eq. 7 is sufficient for inference. In all other cases, the econometrician requires a research design that delivers an approximation of Eq. 5.

\subsection{Research Designs}

There are essentially three research designs in use that approximate the average treatment effect in Eq. 5: crosssectional approaches, use of time-series variation, and a hybrid known as "long differences." The conceptual tradeoffs to these designs center around (i) whether it is reasonable to assume that distinct populations are comparable units after the econometrician has conditioned on observable characteristics and (ii) whether climatic events observed to affect a population are sufficient to capture relevant direct effects and belief effects of climate.

\subsubsection{Cross-sectional approaches}

In cross-sectional research designs, different populations in the same period $\tau$ are compared to one another after conditioning on observables $\mathbf{x}_{i \tau}$. The core assumption needed for this approach is the unit homogeneity assumption as written in Eq. 6. Under this assumption, if different populations have the same climate, then their expected conditional outcomes are assumed to be the same. This allows the econometrician to attribute all differences in observed outcomes to differences in climate, by estimating Eq. 7 having assumed Eq. 6. In a linear framework, this estimate is usually implemented via a regression equation of the form

$$
Y_{i}=\hat{\alpha}+\mathbf{C}_{i} \hat{\beta}_{C S}+\mathbf{x}_{i} \hat{\gamma}+\hat{\epsilon}_{i}
$$

where $\tau$ subscripts are omitted because all observations occur in the same period. Here, $\hat{\alpha}$ is a constant, $\hat{\gamma}$ are effects of observables, and $\epsilon_{i}$ are unexplained variations. The estimate of interest $\hat{\beta}_{C S}$ is a column vector of coefficients describing marginal effects of terms in $\mathbf{C}_{i}$, the set of parameters ${ }^{4}$ selected by the econometrician to characterize the probability distribution of $\mathbf{v}$ at each location $i$.

This design was used widely in early econometric analyses of the effect of the climate (Fankhauser, 1995; Tol, 2009), gaining prominence in the seminal work by Mendelsohn et al. (1994) who regressed farm prices across US counties on growing season temperatures and observable characteristics of farm properties. This implementation highlights a major strength of this approach in the context of climatic effects: since farmers who inhabit a location for a long period will have a strong grasp of $\mathbf{C}$ at their location and will adjust farm investments and management to optimize based on these beliefs, farm prices can be assumed to reflect all direct effects as well as all belief effects. An additional benefit of the cross-sectional research design is that it can be enriched by imposing additional structure on the model and still remains tractable, such as work by Costinot et al. (forthcoming) and Desmet \& Rossi-Hansberg (2015) who consider the effect of climate on the spatial allocation of production, labor, and trade.

A weakness of the cross-sectional approach is its vulnerability to omitted variables bias. When variables that affect $Y_{i}$ are not included in either $\mathbf{C}_{i}$ or $\mathbf{x}_{i}$ but are correlated with one of their elements, the resulting estimates will be biased (Wooldridge, 2002). The surmountability of this problem may be limited because Eq. 6 is untestable, i.e. there exists no systematic method for determining whether any key variables are omitted from Eq. 8 and thus an econometrician can never be certain their model is unbiased.

\footnotetext{
${ }^{4}$ Note that in practice, econometricians must estimate $\hat{\mathbf{C}}$ from data, which is often implemented by estimating moments of $\psi$ using historical data describing $\mathbf{v}$. In principle, $\mathbf{C}$ need not be estimated from real world data, for example it could be constructed using a theoretical or numerical climate model.
} 
One approach designed to address the concern of omitted variables bias is to saturate the model with as many variables as possible. For example, Nordhaus (2006) developed a novel $1^{\circ} \times 1^{\circ}$ gridded global data set of economic production and numerous geographic and climatic factors, which was then applied to Eq. 8 at the pixel level to estimate the effect of temperature on economic productivity. Another approach to constrain the influence of omitted variables is to limit the subsamples of observations for which Eq. 6 is assumed by only comparing populations that are thought to have similar unobservable characteristics. For example, Albouy et al. (2010) estimate the effect of temperature on housing prices across the US, focusing on within-locality comparisons because many characteristics that distinguish localities are difficult to parametrize for inclusion in Eq. 8 but are likely correlated with climatic differences across localities and would thus bias $\hat{\beta}_{C S}$ in a fully pooled regression.

It is not possible to determine that all important variables have been included in Eq. 8, although in some sectors where the data generating process is well known, such as maize yields in the US (Schlenker, 2010), an accumulation of studies may provide us with modest confidence that most important factors are accounted for. Yet in other cases, such as civil wars (Burke et al., 2015b), it is generally assumed that a comprehensive suite of important non-climatic factors may never be known, imposing a ceiling on the assurance we can achieve when using the cross-sectional research design for these outcomes.

\subsubsection{Identification in time-series}

An alternative approach to approximating Eq. 5, instead of assuming populations $i$ and $j$ are comparable, is to examine only population $i$ across separate periods (indexed by $\tau$ ) when different environmental conditions are realized at $i$. This approach conditions outcomes on $\mathbf{c}_{i \tau}$, where each observation summarizes a joint distribution of many vectors $\mathbf{v}_{i t}$ observed during the period $\tau$. An advantage of this approach is that it relies on a plausibly weaker form of the unit homogeneity assumption since it only requires that an individual population $i$ is comparable to itself across moments in time. However, this approach can only approximate Eq. 5 by introducing a second assumption that I call the marginal treatment comparability assumption

$$
\mathrm{E}\left[Y_{i} \mid \mathbf{c}_{\tau}\right]-\mathrm{E}\left[Y_{i} \mid \mathbf{C}_{1}\right]=\mathrm{E}[Y_{i} \mid \mathbf{C}_{1}+\underbrace{\left(\mathbf{c}_{\tau}-\mathbf{C}_{1}\right)}_{\Delta \mathbf{C}}]-\mathrm{E}\left[Y_{i} \mid \mathbf{C}_{1}\right]=\mathrm{E}\left[Y_{i} \mid \mathbf{C}_{2}\right]-\mathrm{E}\left[Y_{i} \mid \mathbf{C}_{1}\right]
$$

where $\mathbf{C}_{2}=\mathbf{C}_{1}+\Delta \mathbf{C}$. This assumption states that the change in expected outcomes between a period where $\mathbf{c}_{\tau}$ is realized relative to outcomes conditioned on a benchmark climate $\mathbf{C}_{1}$ is the same as the change in expected outcomes if the distribution characterized by $\mathbf{C}_{1}$ were distorted by adjustments to climate parameters by $\Delta \mathbf{C}$, defined as the difference between the realized measures $\mathbf{c}_{\tau}$ and the climate values $\mathbf{C}_{1}$, to create a new distribution characterized by $\mathbf{C}_{2}$ (see Figure 1). In other words, marginal treatment comparability assumes that the effect of a marginal change in the distribution of weather is the same as the effect of an analogous marginal change in the climate. Because this assumption has been widely debated, in following subsections I propose a partial test of this assumption and derive some conditions under which it holds exactly.

In a linear framework, this approach is usually implement using either time-series or panel data via a regression equation of the form

$$
Y_{i \tau}=\hat{\alpha}_{i}+\mathbf{c}_{i \tau} \hat{\beta}_{T S}+\mathbf{x}_{i \tau} \hat{\gamma}+\hat{\theta}_{(i)}(\tau)+\hat{\epsilon}_{i}
$$

where $\hat{\alpha}_{i}$ are unit-specific fixed effects that absorb the effect of all time-invariant factors that differ between units,

including unobservables that could not be accounted for in the cross-sectional research design. $\hat{\theta}_{(i)}(\tau)$ are trends in the outcome data, often accounted for using period fixed-effects and/or linear or polynomial time trends, which may be region- or unit-specific.

This approach was probably first proposed by Huntington (1922) who argued, "The ideal way to determine the effect of climate would be to take a given group of people and measure their activity daily for a long period, 

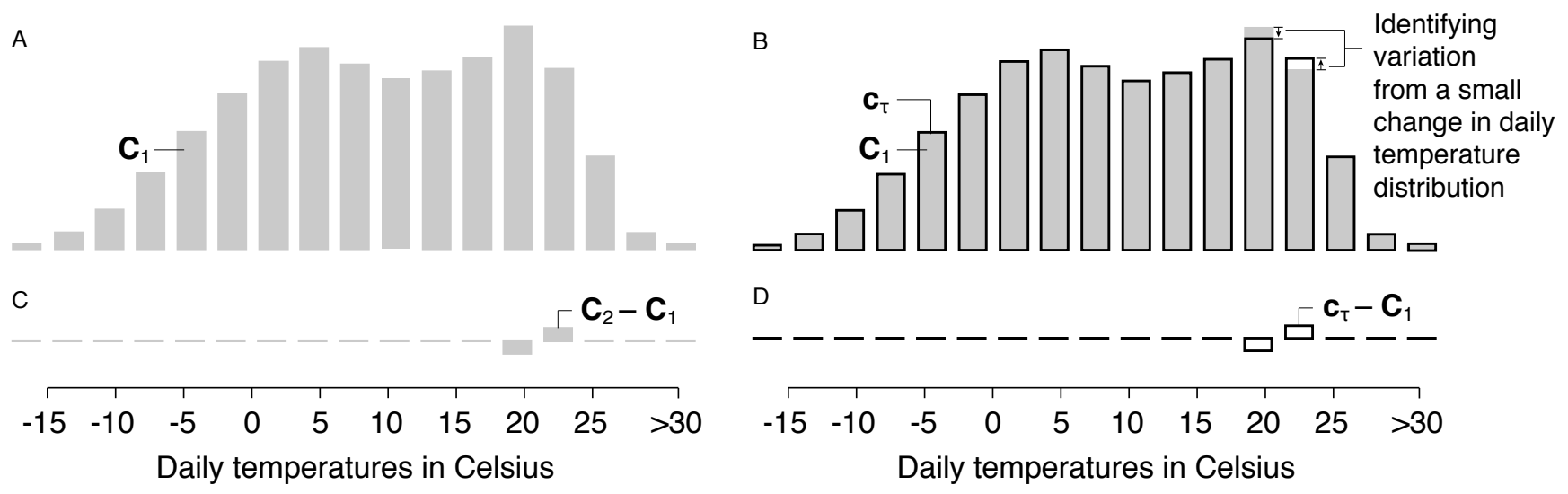

Figure 1: Illustration of the marginal treatment comparability assumption, adapted from Deryugina \& Hsiang (2014). (A) expected annual distribution of daily temperatures for Middlesex county, Massachusetts, a characterization of the climate $\mathbf{C}_{1}$. (B) Black-outlined bars are an example weather summary $\mathbf{c}_{\tau}$ of temperature realizations, in the form of a distribution, during period $\tau$, overlaid on $\mathbf{C}_{1}$. (C) Difference between a climate $\mathbf{C}_{2}$, with structure identical to the realized distribution of weather $\mathbf{c}_{\tau}$ in (B), and the initial climate $\mathbf{C}_{1}$ in (A). (D) Difference between the realized distribution of weather and the climate: $\mathbf{c}_{\tau}-\mathbf{C}_{1}$. The marginal treatment comparability assumption states that the effect of the change in the weather distribution in panel (D) is the same as the effect of the change in the climate distribution in panel $(\mathrm{C})$.

first in one climate, and then in another," and implemented analogs to Eq. 10 using factory-worker data. This approach gained prominence in modern economic analysis when used by Deschênes \& Greenstone (2007), who analyzed whether agricultural profits in US counties responded to "random fluctuations in weather."

The core benefit of this approach is that it accounts for unobservable differences between units, eliminating a potential source of omitted variables bias. However, this approach still remains vulnerable to omitted variables bias if there are important time-vary factors that influence the outcome and are correlated over time with $\mathbf{c}_{i \tau}$ or $\mathbf{x}_{i \tau}$ after conditioning on trends $\theta_{(i)}(\tau)$. It is usually assumed that variations in $\mathbf{c}_{i \tau}$ over time are exogenous to changes in social and economic changes since they are driven by stochastic geophysical processes, but Hsiang (2010) pointed out that many dimensions of $\mathbf{c}_{i \tau}$ are correlated over time because they are partially driven by the same processese.g. temperature, rainfall and hurricanes are all modulated by ENSO - so $\hat{\beta}_{T S}$ may be biased if important climatic variables are omitted. A separate concern raised by Auffhammer et al. (2013) and Hsiang et al. (2015) is that weather data might not be orthogonal to socio-economic conditions because weather-reporting is endogenous. The extent to which these two issues affect the literature as a whole remains unknown.

Some authors introduce time-varying non-climatic factors as controls in Eq. 10, such as crop prices or avoidance behavior, although Hsiang et al. (2013) caution that this may introduce new biases if these factors are endogenous and affected by climatic events, a situation known as "bad control" (Angrist \& Pischke, 2008).

A special case of the time-series research design are cohort analyses, such as Maccini \& Yang (2009) who examined the long-term effects of rainfall during childhood among girls in Indonesia. In these implementations, sequential cohorts within a location $i$ are assumed to be comparable to one another conditioning on $\mathbf{x}_{i \tau}$, differing only in their exposure to sequential realizations of $\mathbf{c}_{i \tau}$. This represents a strengthening of the unit homogeneity assumption, since sequential cohorts within $i$ are different populations that are assumed to be comparable.

\subsubsection{A hybrid approach: long differences}

An approach that aims to compromise between the strengths and weaknesses of cross-sectional analysis and time series identification is the long differences strategy, where changes for both the outcome and the climate within locations are correlated across locations. Long differences is a cross-sectional comparison of changes over time, 
which for two periods of observation $\left\{\tau_{1}, \tau_{2}\right\}$ is implemented with the regression

$$
Y_{i \tau_{2}}-Y_{i \tau_{1}}=\hat{\alpha}+\left(\mathbf{c}_{i \tau_{2}}-\mathbf{c}_{i \tau_{1}}\right) \hat{\beta}_{L D}+\left(\mathbf{x}_{i \tau_{2}}-\mathbf{x}_{i \tau_{1}}\right) \hat{\gamma}+\hat{\epsilon}_{i}
$$

where $\hat{\alpha}$ represents the secular change in $Y$ over time and $\hat{\beta}_{L D}$ represents the extent to which trends in climate are correlated across space with trends in $Y$. This approach is known as "long" differences because it is primarily used to test whether gradual changes in $\mathbf{c}$ induce gradual changes in $Y$, so $\tau_{1}$ and $\tau_{2}$ are usually chosen to be two periods far apart in time. When long-differences has been implemented to measure the effects of climate on growth (Dell et al., 2012), crop yields (Burke \& Emerick, forthcoming; Lobell \& Asner, 2003), and conflict (Burke et al., 2015b), authors have found that $\beta_{L D}$ is almost identical to $\beta_{T S}$, leading them to conclude that gradual changes in $\mathbf{c}$ likely induce similar effects to more rapid changes in $\mathbf{c}$.

The benefit of long differences, relative to time-series analyses that use short differences, is that the marginal treatment comparability assumption in Eq. 9 might be more plausibly satisfied since changes in $\mathbf{c}$ are gradualalthough a weakness of this approach relative to pure cross-section is that some form of this assumption is still required. The benefit of this approach relative to pure cross-sectional analyses is that it requires a weaker form of the unit homogeneity assumption where only changes in $Y$ are assumed comparable across units rather than requiring levels of $Y$ to be comparable, but this assumption remains stronger than the weak within-unit homogeneity assumption required for time-series identification. This tension between the marginal treatment comparability assumption and the unit homogeneity assumption is an overarching challenge to research design in this literature, discussed below.

\subsection{Tradeoff between low-frequency variations and credible identification}

The extent to which Eq. 10 identifies direct effects and belief effects of the climate is often thought to depend on the lengths of periods over which the distribution $\psi\left(\mathbf{c}_{i \tau}\right)$ is summarized, that is $\bar{t}-\underline{t}$. Because belief effects are caused by agents responding to the belief that they face a probability distribution of outcomes described by $\mathbf{C}_{i \tau}$, the extent to which these effects are captured by Eq. 10 likely depends on agent's belief that changes in the distribution of realized measures $\mathbf{c}_{i \tau}$ reflects changes in the prior probability of those events occurring. It is widely assumed that agents facing events $\mathbf{v}_{i t}$ for long $\tau$ will update their beliefs over $\mathbf{C}_{i \tau}$ whereas agents experiencing experiencing events during a short period-perhaps for a period only five minutes long - will not alter their beliefs over $\mathbf{C}_{i \tau}$ for that interval. Thus, while individuals might experience the direct effects of climatic events during short $\tau$, they may be unlikely to alter their beliefs about the climate they face because of a short-lived event.

Because of this logic, it is widely thought that low-frequency data (long $\Delta \tau=\tau_{2}-\tau_{1}=\bar{t}-\underline{t}$ for regularly spaced data) is required to measure belief effects when using time-series variation, since populations only adjust their beliefs if environmental changes are persistent. In the limit that frequencies of $\mathbf{c}_{i \tau}$ exploited by the econometrician approach zero (i.e. the length of $\Delta \tau$ approaches infinity), the research design actually approaches the pure crosssectional analysis in Eq. 8. Thus, the motivation to exploit low-frequency data in time-series designs mirrors the motivation of cross-sectional analysis as they are both thought to capture both direct effects and belief effects of climate changes. Early examples of this approach were Zhang et al. (2007) and Tol \& Wagner (2010), both of whom apply a low-pass filter to climatic variables before estimating Eq. 10. A related alternative approach is to use climate data sampled at a low frequency, implemented by Bai \& Kung (2011) who count droughts over each decade to form each observation in a millenial-scale time series.

While exploiting low-frequency variations in $\mathbf{c}$ is appealing because such an approach might capture both direct and belief effects, it comes at the cost of less credible identification, an issue highlighted by Hsiang \& Burke (2014) 

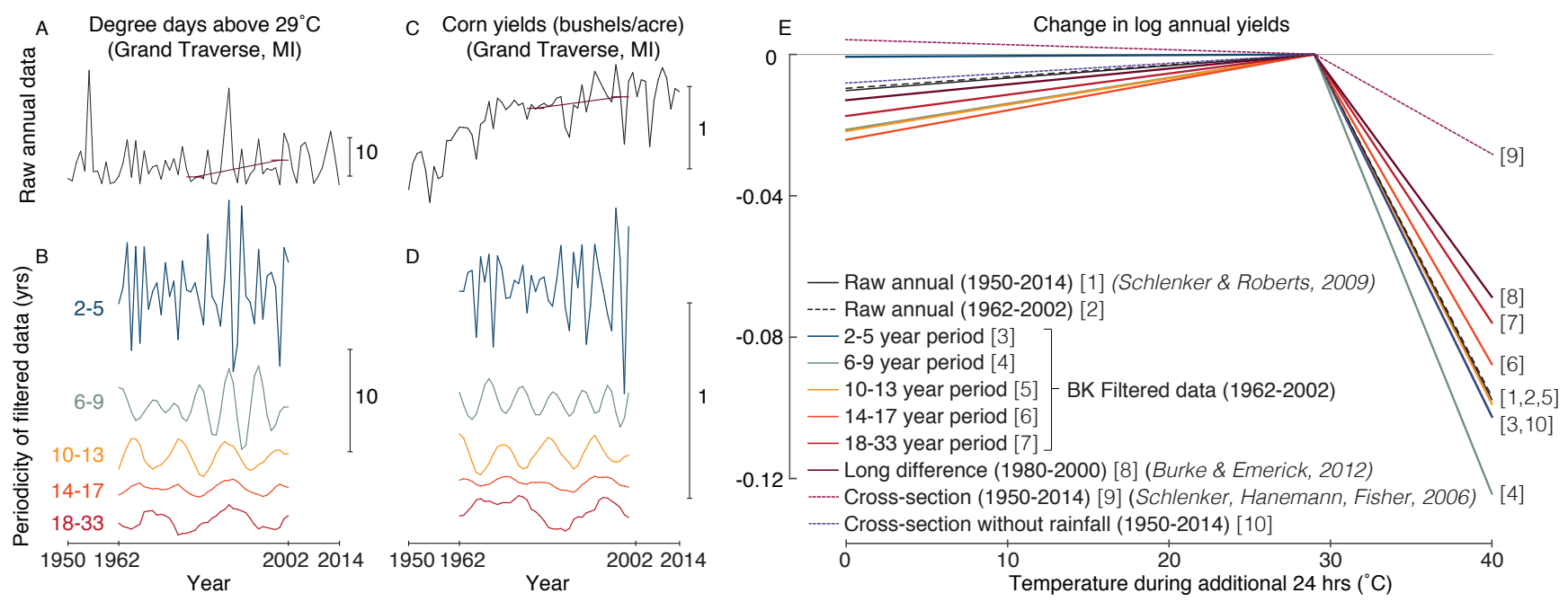

Figure 2: (A)-(D) Example outcome and climate time series data from Grand Traverse, Michigan filtered at different frequencies. (A) raw annual degree-days data (black) and 30 year long-difference (maroon) following Burke \& Emerick (forthcoming). (B) Same data decomposed into time-series at different frequencies, where a Baxter-King band-pass filter has been applied for different periodicities. Filtering causes loss of data at start and end of time series. (C) same as (A) but for corn yields. (D) Same as (B) but for corn yields. Comparison of estimated effect of daily temperature using raw panel data sets, filtered data sets, long differences, and cross-sectional approaches. Sample and estimation indicated by both line and bracketed numbers.

as the frequency-identification tradeoff. The unit homogeneity assumption for time series identification is

$$
\mathrm{E}\left[Y_{i \tau} \mid \mathbf{C}, \mathbf{x}_{i \tau}\right]=\mathrm{E}\left[Y_{i, \tau+\Delta \tau} \mid \mathbf{C}, \mathbf{x}_{i, \tau+\Delta \tau}\right]
$$

where units of observation are assumed to be comparable across periods of observation. However, as the frequency $(1 / \Delta \tau)$ of observation becomes lower, the assumption that $Y_{i \tau}$ and $Y_{i, \tau+\Delta \tau}$ are comparable becomes increasingly difficult to justify. For example, populations separated by multiple centuries might not be comparable units.

The tension between credible identification and use of low-frequency climate variation is not easily resolved if populations do not update their beliefs about the climate more quickly than these populations naturally change in other fundamental ways. In cases where belief effects are large relative to direct effects, then the frequencyidentification tradeoff may represent a major challenge to credible identification of the total effect of the climate. Importantly, however, if the primary way in which belief effects manifest is to alter the direct effects of the climatei.e. belief effects are mostly adaptations designed to cope with direct effects - than total effects of climate still may be nearly identified with high-frequency time-series. Even when this condition is not satisfied, exact identification may still be possible, as shown in Section 2.4.

\subsection{A partial test of marginal treatment comparability}

Unit homogeneity assumptions can be weakened but never tested or eliminated entirely, a fundamental limitation in causal inference generally. However, it may be possible to implement a partial test of the marginal treatment comparability assumption by comparing whether estimated effects are similar when using approaches that exploit climatic variations at different temporal frequencies. If $\hat{\beta}_{C S}=\hat{\beta}_{L D}=\hat{\beta}_{T S}$, i.e. the effects of high frequency changes equal the effects estimated with long differences and in cross-section, then one possible explanation is that the marginal treatment comparability assumption is valid and temporary changes in realizations of $\mathbf{c}$ have similar effects to analogous changes in $\mathbf{C}$. This could be true if the sum of all belief effects are small on net. Versions of these different comparisons were implemented and discussed in Burke \& Emerick (forthcoming); Dell et al. (2009); 
Hsiang \& Jina (2015); Lobell \& Asner (2003); Schlenker \& Roberts (2009) and Burke et al. (2015b), where any difference in estimated effects were attributed to "adaptations" to climate, i.e. belief effects that interact with direct effects. However, a known difficulty is that the strength of this test relies directly on the validity of the different unit homogeneity assumptions used in each of the models compared. It is theoretically possible to obtain $\hat{\beta}_{C S}=\hat{\beta}_{L D}=\hat{\beta}_{T S}$ by chance even if all key assumptions are violated, so long as biases have countervailing effects.

Building on these earlier partial tests, I propose that the credibility of this approach can be further strengthened by estimating climate effects using a spectrum of data that has been filtered at all different temporal frequencies. If the estimated effect of changes in $\mathbf{c}$ is stable across all temporal frequencies spanning from unfiltered time-series data to long-differences and the zero-frequency cross-section, then it seems less plausible that omitted variables biases at different frequencies are exactly offsetting belief effects and more plausible that the marginal treatment comparability assumption is valid. The idea for this test comes from the observation that a time series of the $k$ th element of the vector $\mathbf{c}$ can be decomposed into the Fourier series

$$
\mathbf{c}_{k \tau}=a_{0}^{k}+\sum_{\omega=1}^{\infty}\left[a_{\omega}^{k} \sin (\omega \tau)+b_{\omega}^{k} \cos (\omega \tau)\right]
$$

where $a_{\omega}^{k}$ and $b_{\omega}^{k}$ are constants representing projections onto the basis functions sine and cosine at varying frequencies

$\omega . a_{0}^{k}$ is a constant, analogous to a long-run average (i.e. $\omega=0$ ). Outcome data $Y$ can be similarly decomposed. If we can find appropriate filters that allow us to isolate only certain frequency bands $[\underline{\omega}, \bar{\omega}]$, then we can estimate Eq. 10 using these filtered data and obtain $\hat{\beta}_{T S}^{[\omega, \bar{\omega}]}$, the estimated relationship between climate variables and an outcome at each time scale. As time scales become longer (frequencies lower) then this estimate should continuously approach the long-differences estimate and eventually the cross-sectional estimate if the marginal treatment comparability assumption is valid and these estimates are unbiased.

To demonstrate this test, I obtained panel data on annual county-level maize yield, temperature, and rainfall used in Schlenker \& Roberts (2009), updated to the year 2014 and restricted to the 730 counties east of the 100th meridian that had no missing observations. I then applied a Baxter-King approximate band-pass filter (Baxter \& King, 1999) to all three variables for various frequencies and estimated Eq. 10 with each set of filtered data. Figure 2 shows the effect of temperature on yields at these various time-scales overlaid with estimates of $\hat{\beta}_{T S}$ as in Schlenker \& Roberts (2009), $\hat{\beta}_{L D}$ as in Burke \& Emerick (forthcoming) and $\hat{\beta}_{C S}$ as in Schlenker et al. (2006). In all cases, except the cross-section, these estimated effects are near one another and not statistically different, suggesting that variations in temperature over time have similar effects on maize yields in this context, regardless of the time-scale of these variations. The uniqueness of the cross-sectional estimate could be explained either by belief effects that emerge only at time scales longer than 33 years (the longest time-scaled of filtered data) or omitted variables bias - although the fact that $\hat{\beta}_{C S}$ changes substantially (to more closely resemble time-series estimates) when rainfall terms are omitted highlights the vulnerability of the cross-sectional approach to misspecification. Nonetheless, these results overall appear consistent with an assumption of marginal treatment comparability in this context, at least for time scales shorter than 33 years.

\subsection{Exact identification of climate effects using weather variation}

Why should low and high frequency variations in climatic variables ever provide comparable treatments? It is possible that cross-section, time-series, long difference, and filtered data all provide similar parameter estimates for $\beta$ by chance, such that the above test of marginal treatment comparability paints a misleadingly consistent picture of climate effects and weather effects that are not related. Such critiques, relying on heuristic arguments, are common in the literature. Nonetheless, there is actual theoretical justification for the marginal treatment comparability assumption. In this section I provide a new derivation demonstrating how, under certain conditions, the total effect 
of climate can be exactly recovered using $\hat{\beta}_{T S}$ derived from weather variation. In essence, this result is a combined application of two well known results, the Envelope Theorem and the Gradient Theorem.

The intuition of the result is as follows. Imagine there are two otherwise identical households that are next-door neighbors on a street that runs North-South. The more northern household faces a very slightly different climate because it is very slightly further north. The difference in climate faced by the two households is vanishingly small, but nonzero. These two households have the ability to adapt many dimensions of their daily life to their beliefs about their respective climates and will adopt slightly different behaviors and investments that maximize various outcomes, generating belief effects. However, if we focus on outcomes that are maximized by the households, then the overall net effect caused by these slightly different adaptation decisions is zero because any marginal benefits that the northern household reaps are exactly offset by additional marginal costs (since the household is at a maximum). Therefore, any difference in the optimized outcome between the two households must come from to the direct effects of the slightly different climate and the influence of slightly different beliefs and adaptations between the two households can be ignored. If a weather realization occurs such that the southern household experiences conditions that are slightly different from what they expect and its distribution of weather actually matches the climate of the northern household, then this "weather effect" on the optimized outcome of the southern household must be exactly the same as the cross-sectional difference across the two households in a year when their weather realizations match their respective climates perfectly - since in both cases there is no influence of changing beliefs on the optimized outcome. Stated simply, the marginal effect of the climate on an optimized outcome is exactly the same as the marginal effect of the weather.

Based on this insight, we can trace out a curve describing climate effects between sequential neighbors by watching how optimized outcomes in each household change when that household is confronted by a weather distribution that matches the climate of their immediate next-door neighbor. The integral of these marginal differences between sequential neighbors must then describe how the climate generates larger differences between households that are not adjacent neighbors and experience climates that differ by a non-marginal amount. Importantly, this integration procedure does not assume that individuals do not adjust their beliefs and adapt to their climate. Rather, the marginal effect of such adjustments for marginal climate changes is zero on an optimized outcome, so marginal effects of weather - which do not cause beliefs to change - can be used as a substitute for marginal climate changes in the integration, despite the presence of changing beliefs and adaptations.

To see this result formally, consider an outcome of interest $Y$ that may be affected by the climate $\mathbf{C}$ through its effect on weather realizations $\mathbf{c}$ and actions $\mathbf{b}$, and which is optimized so it can be written as a value function, i.e. the solution to a maximization problem over an outcome-generating function $z(\mathbf{b}, \mathbf{c})$. If we assume $z$ is differentiable and concave in $\mathbf{b}$, then there will be a unique optimum $\mathbf{b}^{*}(\mathbf{C})$ for each climate:

$$
Y(\mathbf{C})=Y\left(\mathbf{b}^{*}(\mathbf{C}), \mathbf{c}(\mathbf{C})\right)=\max _{\mathbf{b} \in R^{N}} z(\mathbf{b}, \mathbf{c}(\mathbf{C}))
$$

recall the notation $\mathbf{c}(\mathbf{C})$ means weather realization $\mathbf{c}$ generated from climate $\mathbf{C}$. Note that maximization of $z$ is allowed to occur through some indirect process, such as efficient market allocations, and need not result from explicit maximization by agents. Figure $3 \mathrm{~A}$ plots the outcome surface $z$ for an example case where $\mathbf{C}$, $\mathbf{c}$, and $\mathbf{b}$ each have only one dimension. For each value of $\mathbf{C}, \mathbf{b}^{*}$ is chosen to maximize $z$ so the outcome $Y$ observed is the locus of optima along the red line.

Let $\mathbf{C}_{1}$ be a benchmark climate at which we are evaluating $Y(\mathbf{C})$. If we differentiate $Y$ by the $k$ th element of C, by the chain rule we have

$$
\frac{\mathrm{d} Y\left(\mathbf{C}_{1}\right)}{\mathrm{d} \mathbf{C}_{k}}=\frac{\partial z\left(\mathbf{b}^{*}\left(\mathbf{C}_{1}\right), \mathbf{c}\left(\mathbf{C}_{1}\right)\right)}{\partial \mathbf{C}_{k}}+\sum_{n=1}^{N} \frac{\partial z\left(\mathbf{b}^{*}\left(\mathbf{C}_{1}\right), \mathbf{c}\left(\mathbf{C}_{1}\right)\right)}{\partial \mathbf{b}_{n}} \frac{\mathrm{d} \mathbf{b}_{n}}{\mathrm{~d} \mathbf{C}_{k}}+\sum_{\kappa=1}^{K} \frac{\partial z\left(\mathbf{b}^{*}\left(\mathbf{C}_{1}\right), \mathbf{c}\left(\mathbf{C}_{1}\right)\right)}{\partial \mathbf{c}_{\kappa}} \frac{\mathrm{d} \mathbf{c}_{\kappa}}{\mathrm{d} \mathbf{C}_{k}}
$$



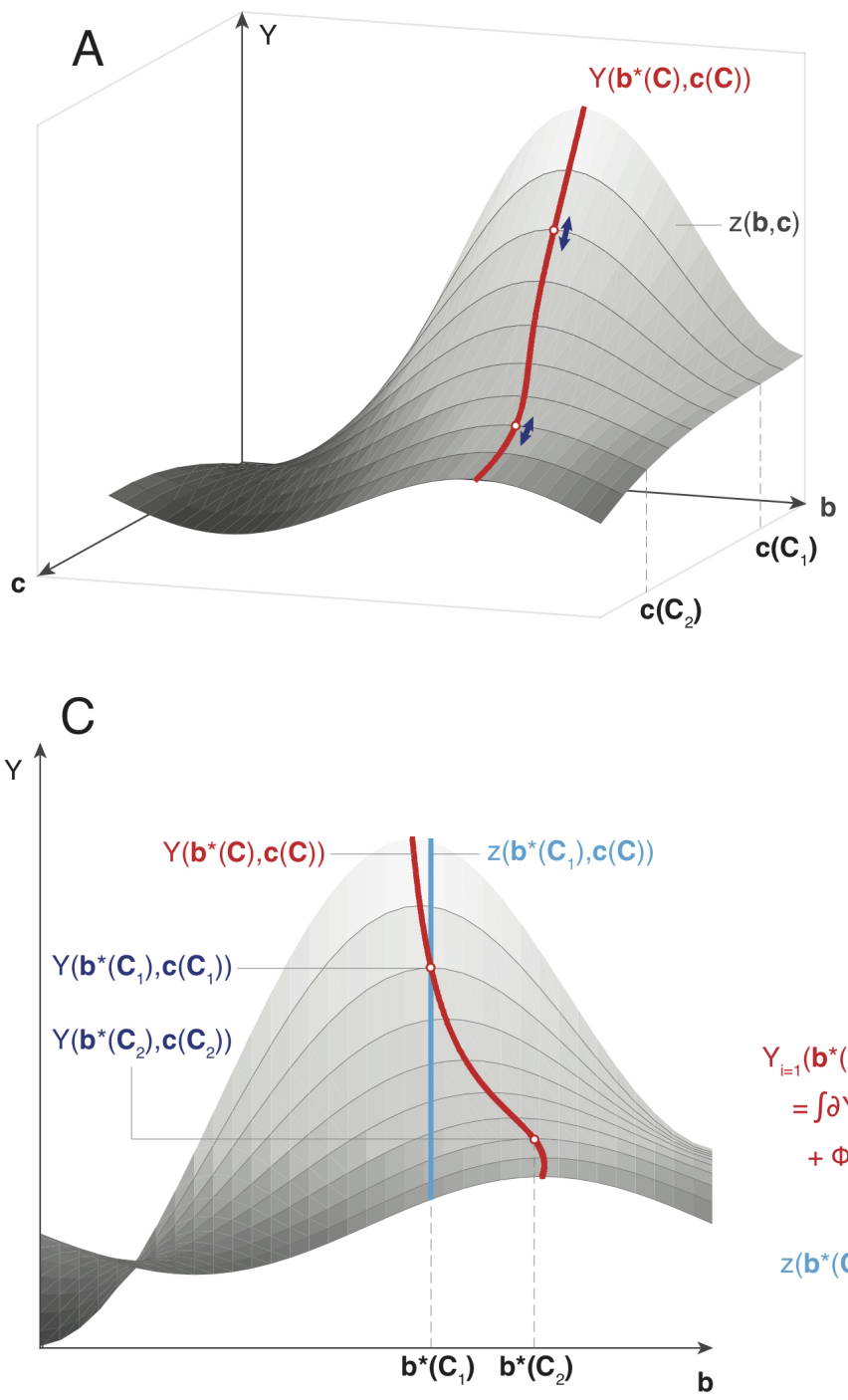

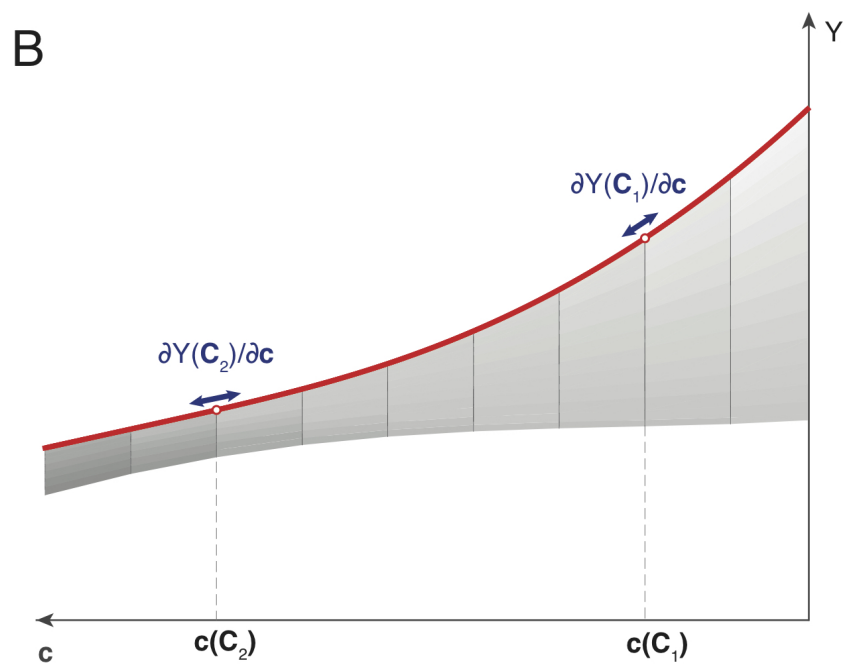

D

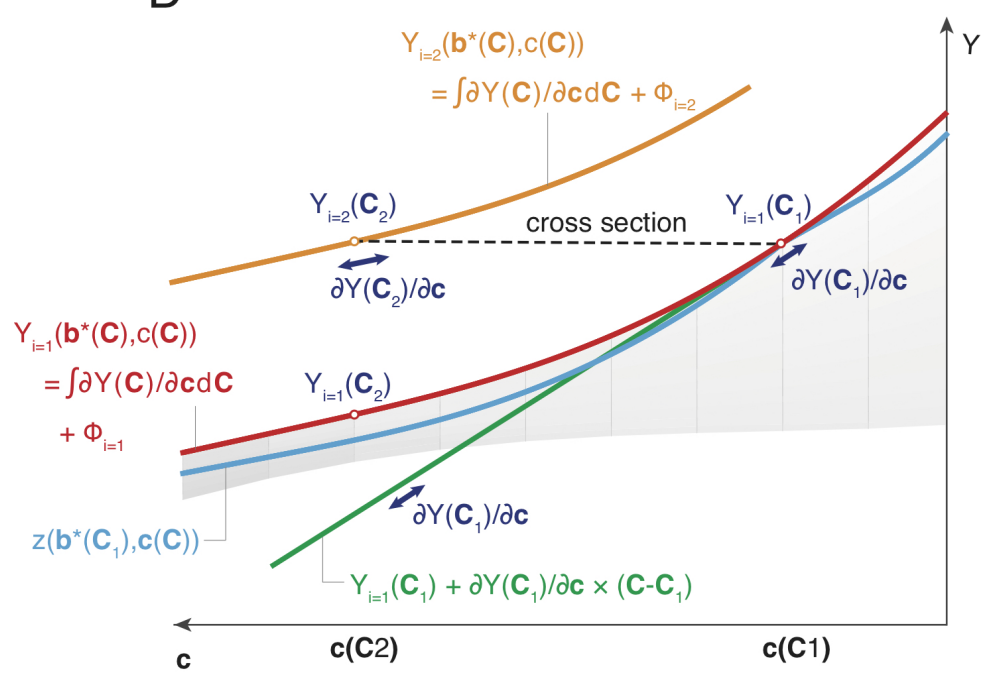

Figure 3: (A) Outcome generating function $z(\mathbf{c}, \mathbf{b})$ over weather outcome $\mathbf{c}(\mathbf{C})$ that reflects the climate and decision variable $\mathbf{b}(\mathbf{C})$ that responds to beliefs about the climate. An implicit fourth dimension not pictured is climate $\mathbf{C}$, where we let $\mathrm{E}[\mathbf{c}(\mathbf{C})]=\mathbf{C}$ for simplicity. Red line is the value function $Y(\mathbf{C})$, the optimum achieved via maximization over $z($.$) , conditional on a given value for \mathbf{c}(\mathbf{C})=\mathbf{C}$, which agents cannot control. (B) Rotated view looking at the $\mathbf{c}-Y$ plane. Local variations in the outcome due to small changes in weather (blue arrows) are tangent to the locus of optima. (C) Rotated view looking at the $\mathbf{b}-Y$ plane. The locus of optima (red line) are achieved because of "adaptation" to changes in climate, indicated by shifts in the $\mathbf{b}$ dimension. If agents beginning at $\mathbf{C}_{1}$ could not adapt, they would be constrained to points on the outcome generating function along the blue line. (D) Same view as (B). Red is $Y(\mathbf{C})$ for location $i=1$. The locus of points along the "no adaptation" blue curve (as in C) lies below the actual optimum for all values except $\mathbf{C}_{1}$. Green is extrapolation of the marginal effect of the climate measured at $\mathbf{C}_{1}$. Orange is $Y(\mathbf{C})$ for location $i=2$, where the integration constant $\phi_{i}$ is different than for $i=1$. Dashed black line is the cross-sectional relationship that would be recovered if $Y_{i=1}\left(\mathbf{C}_{1}\right)$ and $Y_{i=2}\left(\mathbf{C}_{2}\right)$ were the sample. 
where

$$
\frac{\partial z}{\partial \mathbf{C}_{k}}=0
$$

since the climate, as a probability distribution, cannot affect any outcome by a pathway other than through the weather realizations it causes and actions based on beliefs regarding its structure. Because $Y$ is the outcome when $z$ has been optimized through all possible adaptations, and it is differentiable in $\mathbf{b}$, we also know

$$
\frac{\partial z\left(\mathbf{b}^{*}\left(\mathbf{C}_{1}\right), \mathbf{c}\left(\mathbf{C}_{1}\right)\right)}{\partial \mathbf{b}_{n}}=0
$$

for all $N$ dimensions of the action space. Thus Eq. 15 simplifies to

$$
\frac{\mathrm{d} Y\left(\mathbf{C}_{1}\right)}{\mathrm{d} \mathbf{C}_{k}}=\sum_{\kappa=1}^{K} \frac{\partial z\left(\mathbf{b}^{*}\left(\mathbf{C}_{1}\right), \mathbf{c}\left(\mathbf{C}_{1}\right)\right)}{\partial \mathbf{c}_{\kappa}} \frac{\mathrm{d} \mathbf{c}_{\kappa}}{\mathrm{d} \mathbf{C}_{k}}=\sum_{\kappa=1}^{K} \frac{\partial Y\left(\mathbf{C}_{1}\right)}{\partial \mathbf{c}_{\kappa}} \frac{\mathrm{d} \mathbf{c}_{\kappa}}{\mathrm{d} \mathbf{C}_{k}}
$$

Noting that for any marginal change in the distribution of weather, there exists a marginal change in climate that is equal in magnitude and structure such that

$$
\frac{\mathrm{d} \mathbf{c}_{\kappa}}{\mathrm{d} \mathbf{C}_{k}}= \begin{cases}1 & \text { for } \kappa=k \\ 0 & \text { otherwise }\end{cases}
$$

focusing only on these analogous measures of weather and $\operatorname{climate}^{5}$, we have

$$
\frac{\mathrm{d} Y\left(\mathbf{C}_{1}\right)}{\mathrm{d} \mathbf{C}_{k}}=\frac{\partial Y\left(\mathbf{C}_{1}\right)}{\partial \mathbf{c}_{k}}
$$

which says that the total marginal effect of the $k$ th dimension of the climate, evaluated at $\mathbf{C}_{1}$, is equal to the partial derivative of the outcome with respect to the same dimension of weather, also evaluated at $\mathbf{C}_{1}$. Locally, the marginal effect of the climate on $Y$ is identical to the marginal effect of the weather. Eq. 20 implies that Eq. 9, the marginal treatment comparability assumption, holds.

The equivalence between marginal effects of climate and weather can be used to construct estimates for nonmarginal effects of the climate by integrating marginal effects of weather. For an arbitrary climate $\mathbf{C}_{2}$, we know from the Gradient Theorem that we can solve for $Y\left(\mathbf{C}_{2}\right)$ by computing a line integral of the gradient in $Y$ along a continuous path through the $k$-dimensional climate space from $\mathbf{C}_{1} \rightarrow \mathbf{C}_{2}$, starting from $Y\left(\mathbf{C}_{1}\right)$ :

$$
Y\left(\mathbf{C}_{2}\right)=\int_{\mathbf{C}_{1}}^{\mathbf{C}_{2}} \frac{\mathrm{d} Y(\mathbf{C})}{\mathrm{d} \mathbf{C}} \cdot \mathrm{d} \mathbf{C}+\phi=\int_{\mathbf{C}_{1}}^{\mathbf{C}_{2}} \frac{\partial Y(\mathbf{C})}{\partial \mathbf{c}} \cdot \mathrm{d} \mathbf{C}+\phi=\int_{\mathbf{C}_{1}}^{\mathbf{C}_{2}} \nabla_{c} Y(\mathbf{C}) \cdot \mathrm{d} \mathbf{C}+\phi
$$

where the substitution from Eq. 20 is made for each of the $K$ elements of the gradient vector $\nabla_{\mathbf{c}} Y(\mathbf{C})=$ $\left[\frac{\partial Y(\mathbf{C})}{\partial \mathbf{c}_{1}}, \ldots, \frac{\partial Y(\mathbf{C})}{\partial \mathbf{c}_{K}}\right]$. Here, $\phi=Y\left(\mathbf{C}_{1}\right)$ is the constant of integration, which is usually unknown-although, in virtually all applications, changes in $Y$ are the focus of investigation and integration constants are differenced out. The vector of differentials $\nabla_{\mathbf{c}} Y(\mathbf{C})$ describes all the marginal effects of the weather measured "locally" at $\mathbf{C}$, which can be estimated empirically by restricting the sample of observations to those near $\mathbf{C}$ and applying Eq. 10

$$
\nabla_{\mathbf{c}} Y(\mathbf{C})=\left.\hat{\beta}_{T S}\right|_{\mathbf{C}} .
$$

This estimate can then be substituted into Eq. 21 to construct an exactly identified change in $Y$ that occurs as the

\footnotetext{
${ }^{5}$ Focusing only on effects of climate and weather where $\kappa=k$ is consistent with interpreting multiple regression coefficients as causal effects of $\mathbf{C}_{k}$ when other dimensions of $\mathbf{C}$ are fully and simultaneously accounted for.
} 
climate is varied from $\mathbf{C}_{1}$ to $\mathbf{C}_{2}$, in the presence of adaptation adjustments in $\mathbf{b}$, using only time-series estimates:

$$
Y\left(\mathbf{C}_{2}\right)-Y\left(\mathbf{C}_{1}\right)=\left.\int_{\mathbf{C}_{1}}^{\mathbf{C}_{2}} \hat{\beta}_{T S}\right|_{\mathbf{C}} \cdot \mathrm{d} \mathbf{C} .
$$

The difference in outcomes due to a change in the climate is computed by integrating a sequence of of weatherderived marginal effects evaluated at each intermediate value of $\mathbf{C}$. Figure 3B illustrates this integration along the "envelope" of the function $z($.$) , and Figure 3 \mathrm{C}$ demonstrates how the locus of points along this integration allows for all adaptations to climatic changes that occur through adjustment of $\mathbf{b}$, reflecting beliefs that evolve with $\mathbf{C}$. As illustrated in Figure 3D, the integral in Eq. 23 differs from extrapolation of marginal weather effects (green line) or changes along a path on the outcome-generating function $z($.$) where \mathbf{b}$ is held fixed, which would occur if agents were constrained not to adapt (blue curve).

To summarize: If the outcome is a solution to a maximization problem (Eq. 14) for a function $z($.$) that is$ continuous and differentiable in the space of all adaptive actions $\mathbf{b}$, then by application of the Envelope Theorem (Eq. 18) we know that the marginal effect of the climate is exactly the same as the marginal effect of an equally structured change in the weather distribution (Eq. 20), if both are evaluated locally relative to an initial climate. By the Gradient Theorem we know that a sequence of marginal effects of the weather empirically estimated via time-series variation at sequential values of $\mathbf{C}$ can then be integrated to compute the effect of non-marginal climate changes (Eq. 23).

Note that this result does not depend on the nature of individuals' expectations.

It is straightforward to extend this result to cases where the climate exerts direct effects on the outcome by altering a constraint on a maximization problem, rather than entering through arguments to the maximand (MasColell et al., 1995).

The black curve in Figure 3D demonstrates how a cross-sectional regression, as in Equation 8, may produce different results than the integration of weather effects proposed here. Cross-sectional analysis does not difference out the integration constant $\phi$, so if $\phi_{i=1} \neq \phi_{i=2}$ for pairs of observations, then a cross-sectional regression will not recover the red curve. In order for the cross-sectional regressions to recover the effect of $\mathbf{C}$ on $Y$ in this context, we require all of the above assumptions as well as the additional assumption that integration constants are identical:

$$
\frac{\mathrm{d} \phi}{\mathrm{d} i}=0
$$

which implies the strong form of the unit homogeneity assumption that units are comparable in levels conditional on the climate (Eq. 6). Thus the set of assumptions necessary for valid cross-sectional identification in this setting is strictly larger than the set of assumptions required for valid time-series identification.

To my knowledge, the above result has not been previously established and as such, existing empirical papers leveraging weather variation do not explicitly check the assumptions critical to this result: that $Y$ is the solution to a [constrained] maximization, that adaptations $\mathbf{b}$ take on continuous values, and that the maximand function $z($.$) is differentiable in b. Furthermore, many prior studies do not properly compute climate effects via Eq. 23,$ with the notable exception of Schlenker et al. (2013) and Houser et al. (2015), who essentially implement a form of this approach explicitly. Total effects of climatic changes in Eq. 23 are also computed correctly in studies where marginal effects of weather are allowed to change based on underlying climatic conditions, and these evolving marginal weather effects are integrated to compute the cost of shifting climatic conditions, as in Hsiang \& Narita (2012) and Burke et al. (2015c). Finally, those studies where the marginal effects of weather are approximately invariant in climate, such as Ranson (2014) and Deryugina \& Hsiang (2014), also basically estimate Eq. 23 when they linearly extrapolate weather effects since the two calculations are equivalent. 

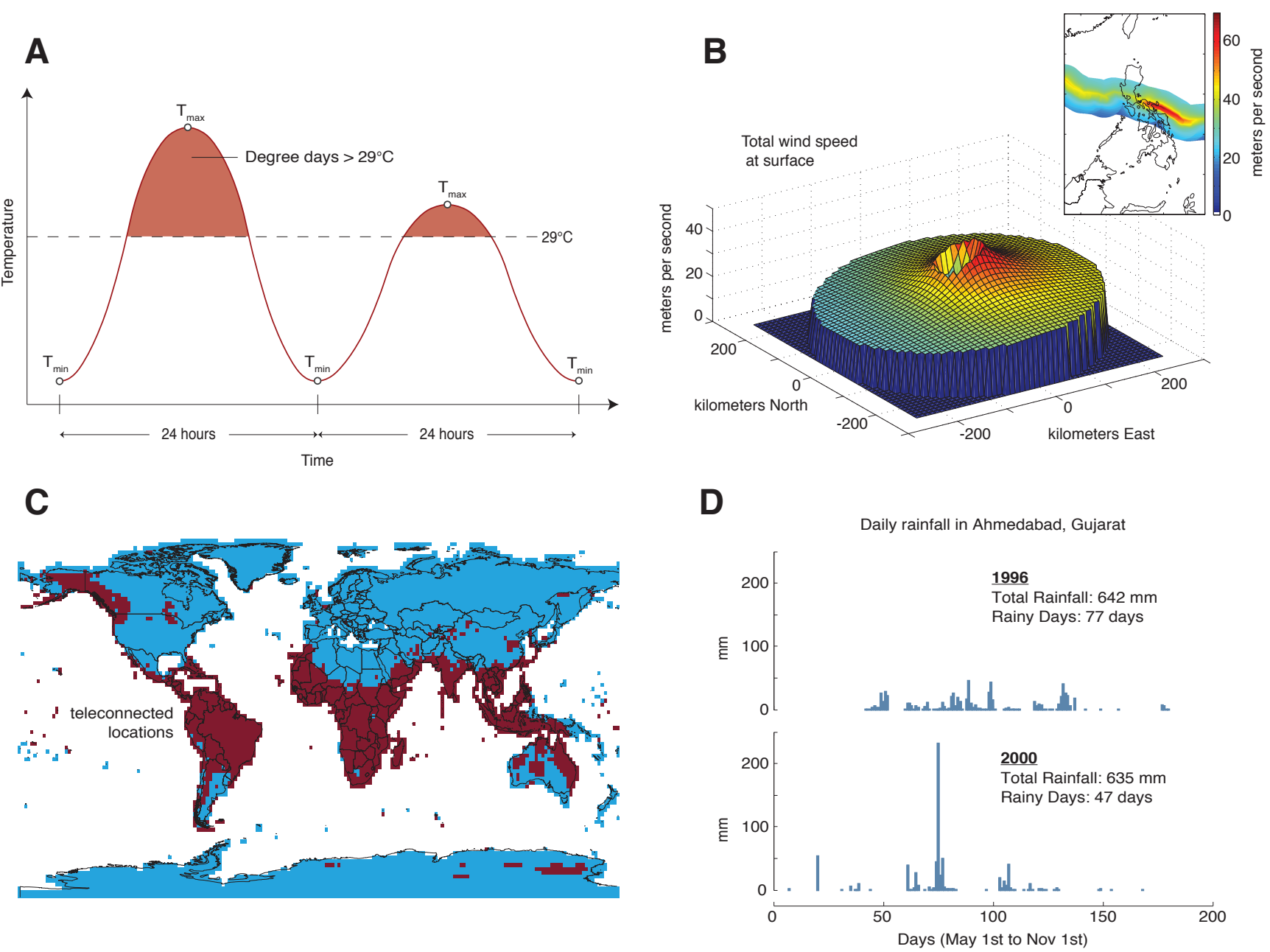

Figure 4: Examples of innovations in climate measurement. (A) Construction of degree days measures using hourly temperature data interpolated between daily minimum and maximum temperature in Schlenker \& Roberts (2009). (B) Wind field model used to reconstruct wind exposure along the path of tropical cyclones (inset is computed exposure of super typhoon Joan) in Hsiang \& Jina (2014). (C) Identification of "teleconnected" pixels (red) that have temperature and rainfall strongly coupled to the El Niño-Southern Oscillation in Hsiang et al. (2011). (D) Example rainfall distributions used to construct "rainy days" count as a measure of within-year rainfall dispersion across two years with similar total rainfall in Fishman (2016).

\section{MEASUREMENT OF CLIMATE VARIABLES}

The measurement of climate variables is a critical methodological step in identifying climate effects, regardless of the research design used. Early analyses concerned only with measuring whether climatic factors had a non-zero effect, or the sign of an effect, used simple measures of climate such as "latitude" or a single indicator variable that is one if a population is exposed to a predefined event (e.g. a drought) and zero otherwise. This approach is internally valid, but has important limitations often under-appreciated in the literature. First, coarse climate measures introduce large measurement errors that will cause attenuation bias, leading to under-rejection of the null hypothesis. Second, the structure of a dose-response function

$$
\mathrm{E}[Y \mid \mathbf{c}]=f(\mathbf{c})
$$


is often of interest, for example we may be interested in nonlinearities or whether multiple dimensions of climate interact in important ways, requiring measures of climate variables be near continuous and multidimensional. Third, if measures of $\mathbf{c}$ do not reflect scalable physical quantities in the real world, we may have little confidence that estimated effects are externally valid to other locations or to periods when the climate may change, for example it is impossible to consider how cyclone intensification may affect outcomes if cyclone exposure is measured only as binary variable. Fourth, pooling a sample of different locations may provide a valid average treatment effect of climatic conditions on the sample, but it may be a poor predictor of outcomes at any actual locations if the physical properties of events coded as similar are not actually physically similar. Finally, the result derived in the previous section, that time-series variations can be used to exactly identify marginal effects of the climate, can only hold if climatic variations are measured in such a way that an econometrician can identify marginal effects - for example, binary treatments are not differentiable and so it may be difficult to determine if changing from "no treatment" to "treatment" is a marginal change.

For all of the above reasons, many of the major innovations in the literature over the past decade have resulted from improvements in the measurement of climate variables, contributing at least as much to recent advances, if not more, than functional form innovations (discussed in the next section). For example, using spatial interpolation techniques, Schlenker \& Roberts (2009) developed estimates of temperature with high spatial and temporal resolution, allowing them to construct precise measures of "degree-days" that integrate cumulative exposure to specific temperature ranges (Fig 4A). Deschênes \& Greenstone (2011) introduced a related approach where days are counted based on their average temperature ${ }^{6}$. Yang (2008) estimated the effect of tropical cyclones by coding a storms' maximum windspeed at landfall, an approach enriched further by Nordhaus (2010) and Mendelsohn et al. (2012) who use additional landfall statistics; Hsiang (2010) expanded measurement of cyclone exposure by integrating wind speed exposure at all points throughout the lifetime of a storm (Fig 4B). Guiteras et al. (2015) implemented a novel technique for detecting surface flooding using satellite imagery. Auffhammer et al. (2006) used an atmospheric circulation model to estimate overhead aerosol exposure. Hsiang et al. (2011) developed a method to identify the ENSO exposure of countries (Fig 4C). Fishman (2016) utilized several metrics to characterize the evenness of rainfall distributions that are similar in total rainfall (Fig 4D). In several cases, researchers find that established linear or nonlinear transformations of fundamental climatic measures, such as temperature, rainfall, and humidity, are useful in explaining patterns of outcomes, such as standardized precipitation evapotranspiration index (Harari \& La Ferrara, 2013), drought indices (Couttenier \& Soubeyran, 2014), vapor pressure deficit (Urban et al., 2015), heat indices Baylis (2015), or malaria ecology indices McCord (forthcoming). In all cases, these various measures can be understood as approaches to collapsing the dimensionality of $\mathbf{c}$ in a manner that efficiently describes patterns that matter from an economic or social standpoint. In most of these cases, alternative approaches to measuring climate variables cannot be viewed as objectively wrong, rather there are many ways of describing data in $\mathbf{c}$ that do not efficiently describe those components of variation that most strongly influence the outcomes of interest. Blunt climate measures are not wrong, they just introduce large measurement errors.

Particular caution is needed when applying the natural logarithm transformation, standard in many economic applications, to climate measures since it is not always sensible. For example, using log(temperature) in Eq. 10 is challenging to interpret because a $1 \%$ change in temperature - used in the interpretation of the resulting coefficients - has different meaning depending on whether temperature is measured in Fahrenheit, Celsius, or Kelvin. In other cases, such transformed data can be fit to a model but the standard interpretation is inconsistent with physical phenomena, for example Nordhaus (2010) and Mendelsohn et al. (2012) model hurricane damage using $\log$ (windspeed $)$ and conclude that damage is "super-elastic" because it appears to grow up to six exponents faster than the energy of the storm - a misinterpretation that is readily reconciled with physics when the log transformation is simply not applied (Camargo \& Hsiang (2016)).

\footnotetext{
${ }^{6}$ See Deryugina \& Hsiang (2014) for a derivation of this approach.
} 
Many dimensions of the climate, such as persistent drought and sea level, remain poorly captured in econometric models due to measurement challenges and future innovations will further improve our understanding of these climate effects substantially.

\section{ECONOMETRIC MODELS}

Having selected a research design and constructed appropriate climate measures, an econometrician must select a model that is fit to the data. Here I discuss five aspects of modeling that have been particularly important in measurement of climate effects: nonlinearities, displacement, uncertainty, adaptation, and cross-study comparisons.

The discussion here is focused on the measurement of climate effects applying a reduced form approach to construct a dose-response surface. Such an approach does not necessarily specify a single pathway through which the climate affects social outcomes, and in many cases it is likely that several pathways play a role. Hsiang et al. (2013) suggest that in order to reject potential pathways in any given context, researchers must look for natural experiments in which a particular pathway is obstructed due to external factors and then examine whether reduced form effects persist - Sarsons (2015) and Fetzer (2014) are useful examples of this strategy.

It is worth noting that a large number of studies in economics utilize variation in weather as an instrumental variable to study the effect of an intermediary variable on an outcome. This strategy relies on the assumption of an exclusion restriction, i.e. the employed weather variation only affects the outcome through the specified intermediary variable. This assumption is untestable, although the large number of studies utilizing exogenous variation in weather to study a large number of outcomes through various proposed pathways seems itself evidence that this assumption cannot be true in many cases.

\subsection{Nonlinear effects}

The interpretation of nonlinear effects, and their estimation, depends heavily on whether observations are highly resolved in space and time or whether they are highly aggregated. Because weather data are often available at high resolution, even when outcome data is not, it is often possible to recover micro-level response functions, below the level of aggregation in the outcome data, by carefully considering the data generating process.

\subsubsection{Recovering local, micro-level, and instantaneous nonlinear effects}

Local effects of climatic variables are often nonlinear in important ways, such as extreme cold days and extreme heat days generating excess mortality (Deschênes \& Greenstone, 2011) or extreme heat hours causing damage to crop yields (Schlenker \& Roberts, 2009). In some cases, such as Graff Zivin \& Neidell (2014) and Aroonruengsawat \& Auffhammer (2011), outcomes are measured at the same daily frequency as these nonlinear effects manifest, rendering their measurement straightforward using standard techniques. However, in most cases nonlinear effects manifest over time-scales (e.g. hours) and spatial scales (e.g. pixels) that are much finer than the periodicity and spatial scale at which outcome data is measured (e.g. annually by country). Similarly, local effects may differ between multiple locations within a unit of observation. Despite aggregation of the outcome across space and over moments in time, it is possible to recover nonlinear relationships at the spatial and temporal scale at which climatic data is recorded. Suppose outcome $Y_{i \tau}$ is observed over regions $i$ (e.g. provinces) made up of more finely resolved positions $s$ (e.g. pixels) during intervals of time $\tau$ (e.g. years) made up of shorter moments $t$ (e.g. days). Let the "instantaneous" nonlinear effect of climate at a moment and position be $f\left(\mathbf{c}_{s t}\right)$, which we approximate as a linear combination of $M$ simple nonlinear functions (e.g. polynomial terms)

$$
f\left(\mathbf{c}_{s t}\right) \approx \beta_{1} f_{1}\left(\mathbf{c}_{s t}\right)+\beta_{2} f_{2}\left(\mathbf{c}_{s t}\right)+\ldots+\beta_{M} f_{M}\left(\mathbf{c}_{s t}\right)=\sum_{m=1}^{M} \beta_{m} f_{m}\left(\mathbf{c}_{s t}\right)
$$




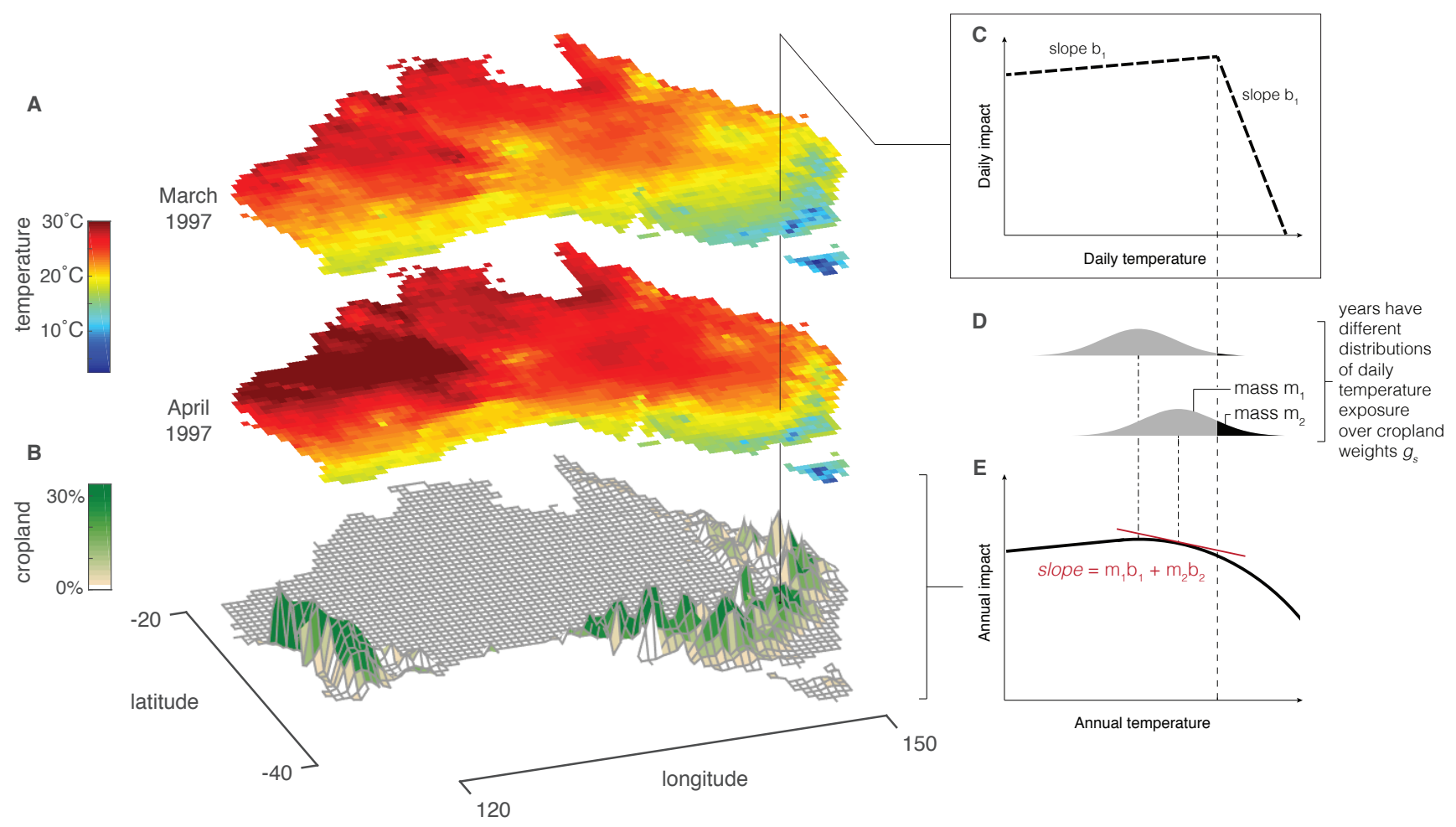

Figure 5: (A) Heterogenous temperatures across locations $s$ within a region are aggregated based on the distribution of units of analysis $g_{s}$, in this case the spatial distribution of croplands (B). This aggregation means that if climate affects outcomes at a highly localized and level (C), shifts in the regional distribution of climatic exposure of $g_{s}$ (D) will generate an aggregate response to aggregated climate measures that is generally smoother (E). Adapted from Burke et al. (2015c).

where the $\beta$ 's are constant coefficients. In practice, $f($.$) has been successfully modeled as an M$-piecewise linear function-as in degree-day models-or an $M$ th order polynomial or restricted cubic spline (Miller et al., 2008; Schlenker \& Roberts, 2009) or interactions between multiple climate measures (Urban et al., 2015), options which have efficiency and (local) differentiability benefits, or a $M$-piecewise constant or "binned" function (Deryugina \& Hsiang, 2014; Deschênes \& Greenstone, 2011), a flexible nonparametric option.

Under the assumption of temporal and spatial separability, i.e. that the outcome of interest is a linear sum of $f\left(\right.$.) across positions and moments, weighted by the number of affected economic units $g_{s}$ (e.g. crop fields) at those positions, then the regressions in Eqs. 8, 10 and 11 are modified to the form

$$
Y_{i \tau}=\alpha_{i}+\left[\sum_{s \in i} \sum_{t \in \tau} \beta_{m} f\left(\mathbf{c}_{s t}\right) g_{s}\right]+\mathbf{x}_{i \tau} \gamma+\theta_{(i)}(\tau)+\epsilon_{i \tau}
$$

where the index and functions of $\tau$ and region effects $\alpha_{i}$ are omitted in the cross-sectional case. Notably, as demonstrated in Welch et al. (2010), the structure of $f($.$) may differ between sub-periods in \tau$ so that Eq. 27 becomes

$$
Y_{i \tau}=\alpha_{i}+\left[\sum_{s \in i}\left(\sum_{t \in \tau_{a}} \beta^{a} f^{a}\left(\mathbf{c}_{s t}\right) g_{s}+\sum_{t \in \tau_{b}} \beta^{b} f^{b}\left(\mathbf{c}_{s t}\right) g_{s}\right)\right]+\mathbf{x}_{i \tau} \gamma+\theta_{(i)}(\tau)+\epsilon_{i \tau}
$$

if $\tau_{a}$ and $\tau_{b}$ represent a partition of period $\tau$. Focusing on Eq. 27 for simplicity, we can substitute the approximation 
from Eq. 26 and interchange the order of summation to obtain

$$
\begin{aligned}
Y_{i \tau} & \approx \alpha_{i}+\left[\sum_{s \in i} \sum_{t \in \tau}\left(\sum_{m=1}^{M} \beta_{m} f_{m}\left(\mathbf{c}_{s t}\right) g_{s}\right)\right]+\mathbf{x}_{i \tau} \gamma+\theta_{(i)}(\tau)+\epsilon_{i \tau} \\
& =\alpha_{i}+\sum_{m=1}^{M} \beta_{m} \underbrace{\left[\sum_{s \in i} \sum_{t \in \tau} f_{m}\left(\mathbf{c}_{s t}\right) g_{s}\right]}_{\tilde{f}_{m i \tau}}+\mathbf{x}_{i \tau} \gamma+\theta_{(i)}(\tau)+\epsilon_{i \tau} \\
& =\alpha_{i}+\sum_{m=1}^{M} \beta_{m} \tilde{f}_{m i \tau}+\mathbf{x}_{i \tau} \gamma+\theta_{(i)}(\tau)+\epsilon_{i \tau}
\end{aligned}
$$

which can be estimated with a linear regression using data at the region-period $(i \tau)$ level. Note that the regressors $\tilde{f}_{m i \tau}$ are weighted sums across space and time of the $m$ th nonlinear function evaluated at locations $s$ and moments $t$ that are not resolved in the outcome data. Estimation of Eq. 29 via regression recovers estimates for $\beta_{m}$ describing the local and instantaneous function $f($.$) , even though it uses coarser data.$

\subsubsection{Nonlinearity in regional summary measures due to local nonlinearities}

Many analyses do not estimate Eq. 29 but instead examine whether nonlinear relationships exist between summary statistics of climate data and aggregated outcome data, usually because constructing $\tilde{f}$ involves highly disaggregated climate data and is therefore challenging. The most common summary statistic of $\mathbf{c}_{k i \tau}$, the $k$ th element in $\mathbf{c}_{i \tau}$, is an weighted average value over region $i$ and period $\tau$

$$
\overline{\mathbf{c}_{k i \tau}}=\sum_{s \in i} \sum_{t \in \tau} \mathbf{c}_{k s t} g_{s}
$$

for example, Dell et al. (2012) construct measures of population-weighted average temperature over entire countries during an entire year. These region-by-period summary statistics may then be used to construct regressors in a nonlinear model, such as the $Q$-order polynomial

$$
Y_{i \tau}=\hat{\alpha}_{i}+\sum_{q=1}^{Q} \hat{\beta}_{q}\left(\overline{\mathbf{c}_{k i \tau}}\right)^{q}+\mathbf{x}_{i \tau} \hat{\gamma}+\hat{\theta}_{(i)}(\tau)+\hat{\epsilon}_{i \tau}
$$

a widely used approach. Eq. 31 differs from Eq. 29 such that the two approaches should not recover identical coefficients, even if the micro-level nonlinear data generating process is unchanged. Burke et al. (2015c) demonstrated that the marginal effects recovered in Eq. 31 should equal the weighted-average marginal effect at the local level (as estimated in Eq. 29), averaged across locations and moments, that are associated with a one unit shift in the distribution of local climatic conditions (Figure 5). Importantly, it is the spatial covariance between weights $g_{s}$ and climatic conditions within periods of observation that determines how local nonlinear effects appear in region-level models like Eq. 31. In general, a wider dispersion of conditions experienced across locations and moments within a summarized region leads to greater smoothing and flattening of the response in Eq. 31 relative to the local instantaneous response (Figure 5C-E). Thus, we expect that larger and more heterogenous regions with

longer periods of observation should produce smoother and flatter responses to summary climate measures, even if local nonlinear effects are unchanged.

\subsubsection{Global nonlinear effects}

The distribution of climatic conditions experienced over time within one region often differs substantially from distributions in other regions. In these cases, marginal effects should differ if response functions are nonlinear. 
A
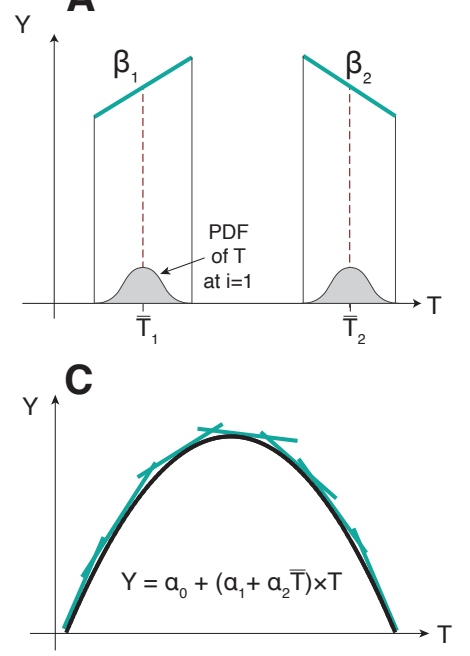

B
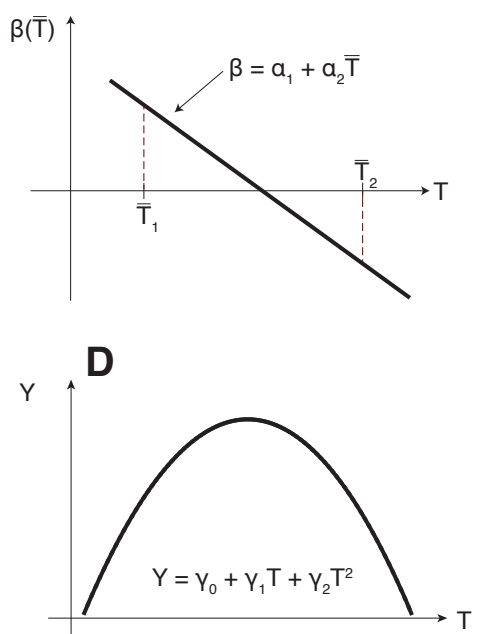

Figure 6: Different marginal effects $\beta$ estimated from variation within different locations with different average climates (A) may result from an interaction with average climatic conditions (B). In a panel data setting, this can be modeled using an interaction (C) where each panel unit is a local linearization of a nonlinear function, or a global nonlinear function can be estimated using the full sample (D).

Marginal effects that change as a function of mean climate conditions are easily modeled as an interaction between average climatic conditions and realizations of climatic variables such that

$$
\frac{\partial Y_{i \tau}}{\partial \mathbf{c}_{i \tau}}=\beta\left(\overline{\mathbf{c}}_{i}\right)
$$

as illustrated in Figure 6A-B. Should an underlying global nonlinear response exist, it can be recovered by estimating a single model that is nonlinear in climate variable realizations, with a response surface that is only locally identified by the time-series variation among units that experience realizations in the neighborhood of a tangency point. This is conceptually analogous to integrating Eq. 32 to recover the global response surface (Figure 6C-D), which holds exactly as $\lim \mathbf{c}_{i \tau} \rightarrow \overline{\mathbf{c}}_{i}$.

\subsection{Displacement and delay}

In many contexts, it is plausible that climatic events at moments in the past or at nearby locations affect an outcome at a specific time and place, much like the surface of a pond observed at any moment and location might depend on whether a raindrop disturbed that location moments before, or a nearby point on the pond surface. When using time-series identification of climate effects, it is crucial to account for these "ripple" effects so that a local transient response is not mischaracterized as a persistent effect. Of particular concern is whether climatic events have a net effect on outcomes, or whether they simply displace outcomes across time and/or space.

So far, we have only considered contemporaneous effects of the vector $\mathbf{c}_{i \tau}$ on outcome $Y_{i \tau}$. We now consider the influence of the entire vector field $\mathbf{c}(s, t)$ defined across all positions $s$ and moments $t$ on the outcome $Y_{i \tau}$.

\subsubsection{Temporal displacement}

A climatic event at time $t$ might bring an event that would otherwise occur at time $t+1$ forward in time, an effect known as temporal displacement or "harvesting." For example, Deschênes \& Moretti (2009) highlighted the importance of this concept by demonstrating that many deaths which occur during hot days in the United States would have likely occurred within the subsequent two months even in the absence of a hot day - they thus conclude 

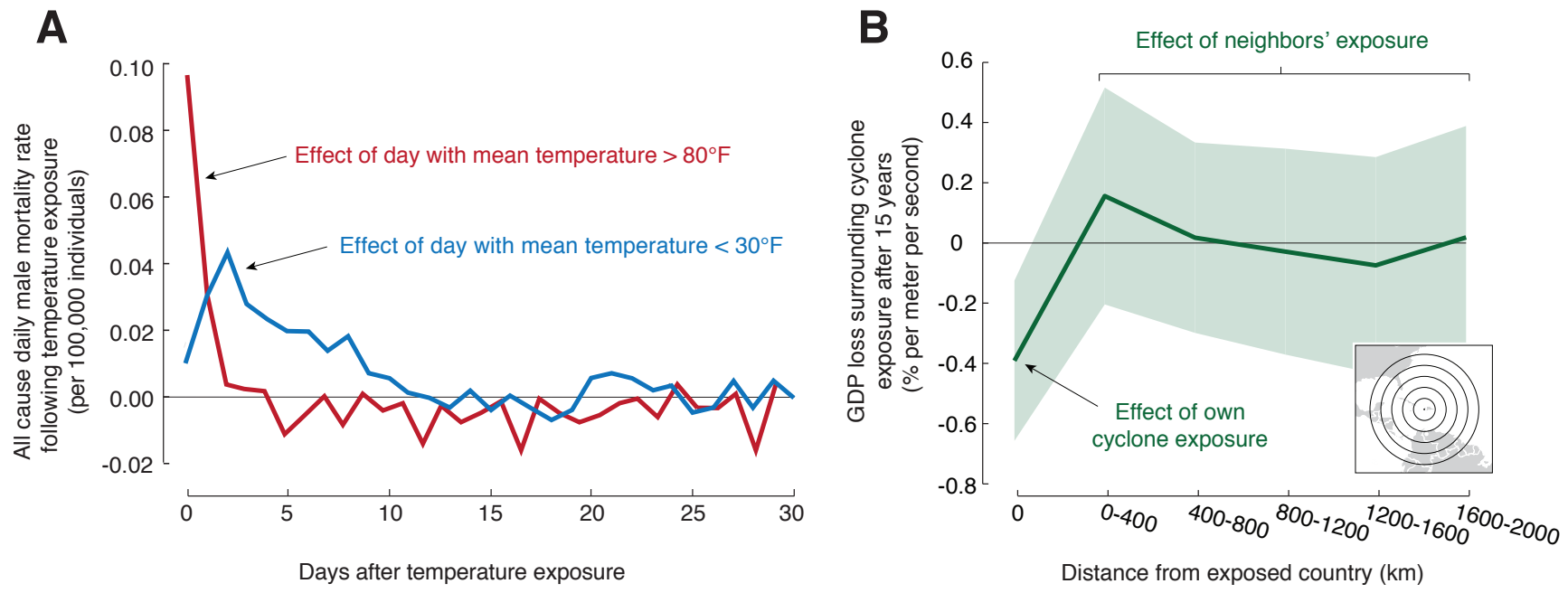

Figure 7: (A) Temporal lag effects of hot and cold days on all cause male mortality; from Deschênes \& Moretti (2009). (B) Spatial lag effects of tropical cyclones on own and neighbor's GDP fifteen years after exposure, inset are example annuli used to construct spatial lags around Haiti; from Hsiang \& Jina (2014).

an effect of a heat wave is to influence the timing of deaths within a relatively narrow window, not only to create entirely "new" deaths (Figure 7A). Mathematically, the signature of temporal displacement is for periods following a climatic event to have a response that is opposite in sign to the contemporaneous response. A challenge to identifying these lagged effects is that the climatic histories of sequential moments overlap, so it may not be the case that outcomes at any moment are only a response to a single historical climate event. Rather, outcomes at each moment represent a superposition of many historical events each at different moments in time. This issue can be resolved by conditioning expected outcomes on the complete history of climatic events using a distributed lag model:

$$
Y_{i \tau}=\hat{\alpha}_{i}+\sum_{l=0}^{L}\left(\mathbf{c}_{i, \tau-l} \hat{\beta}_{l}\right)+\mathbf{x}_{i \tau} \hat{\gamma}+\hat{\theta}_{(i)}(\tau)+\hat{\epsilon}_{i}
$$

where $l$ is a lag length measured in periods ( $l=0$ indicates a contemporaneous observation) and the maximum lag length considered is $L$. The identifying assumption to this approach is that the influence of a climate event at $\tau_{0}$ on outcomes at $\tau_{1}$ is determined by the length of time $\tau_{1}-\tau_{0}$ separating the observations. As written, this model also assumes additive separability between lagged effects, although this assumption can be relaxed by interacting lagged terms. It is somewhat standard in the literature to sometimes include negative lags (leads) in Eq. 33 as a falsification exercise, since it generally assumed that future climatic events do not effect outcomes substantially.

The net effect of a one-unit climatic event after $\lambda$ periods is the cumulative effect

$$
\hat{\Omega}_{\lambda}=\sum_{l=0}^{\lambda} \hat{\beta}_{l} .
$$

If all of the effects of a climate event are to displace outcomes in time, then $\hat{\Omega}_{\lambda=L}$ will be zero, whereas a positive or negative cumulative effect indicates that climatic events caused additional changes beyond altering the timing of events. It is worth noting that when the outcome is a growth rate, than these cumulative effects represent changes in levels, as explored and discussed by Dell et al. (2012) and Hsiang \& Jina (2014). Burke et al. (2015c) compute $\Omega_{\lambda}$ in a nonlinear context. 


\subsubsection{Delayed effects}

Eq. 33 is also used to detect delayed effects, which may arise even if contemporaneous effects $\left(\hat{\beta}_{l=0}\right)$ are small or zero but lagged effects $\left(\hat{\beta}_{l>0}\right)$ are large. In several cases, such as the effect of cold days on mortality (Fig. 7A) (Deschênes \& Moretti, 2009) or the effect of tropical cyclones on employment and income (Anttila-Hughes \& Hsiang, 2012; Deryugina, 2015), delayed effects are of first-order relevance, dominating contemporaneous effects.

\subsubsection{Spatial displacement and remote effects}

Similar to temporal displacement and delay, it is possible that climatic events cause outcomes to be displaced across space or to trigger remote outcomes (analogous to "delayed effects in space") even if local effects are limited, perhaps because markets and price signals efficiently transmit the influence of the climate across locations. For example, Hsiang \& Jina (2014) examine whether cyclone strikes displace income growth to nearby countries (Figure 7B). The econometric challenge associated with identifying these effects is analogous to the temporal case as overlapping spatial effects may complicate the spatial distribution of outcomes, similar to multiple simultaneous raindrops generating overlapping rings of waves in a pond. The solution is also similar and involves estimating a spatial lag model analogous to Eq. 33, but where lags are applied to the index $i$, rather than $\tau$, based on the distance between contemporaneous observations and effects at all distances are estimated simultaneously. Similar to temporal lags, the net effect of a climatic event can be considered by summing lags, although care must be taken as the number of observations at varying distances may not necessarily by constrained and will depend on the spatial arrangement of units. This approach performs especially well when it is applied to data on a regular grid, as demonstrated by Harari \& La Ferrara (2013). In cases where remote effects may be delayed, than a model with spatial-temporal lags is required:

$$
Y_{i \tau}=\hat{\alpha}_{i}+\sum_{l=0}^{L} \sum_{\pi=0}^{\Pi}\left(\overline{\mathbf{c}}_{\{j \mid D(i, j)=\pi\}, \tau-l} \hat{\beta}_{l \pi}\right)+\mathbf{x}_{i \tau} \hat{\gamma}+\hat{\theta}_{(i)}(\tau)+\hat{\epsilon}_{i}
$$

where $\overline{\mathbf{c}}_{\{j \mid D(i, j)=\pi\}, \tau-l}$ is the average climate exposure of all locations $j$ that are a distance $\pi$ to location $i$ (where the outcome is observed) at time $\tau-l$. For example, Figure 7B displays the cumulative growth effect of a cyclone as a function of distance from the event.

\subsection{Statistical uncertainty}

Uncertainty estimates for regressions must account for the strong spatial and temporal autocorrelation in climatic exposure, regardless of the research design employed. The concern is that unobservable omitted variables may also be autocorrelated, such that spurious correlations with climate events occur with greater frequency than if all observations were independently distributed - this will cause bias in estimates of standard errors even though estimated climate effects $\hat{\beta}$ may be unbiased (Bertrand et al., 2004; Moulton, 1986). The extent of the bias in standard errors depends on the spatial scale and sampling frequency of the data relative to natural patterns of autocorrelation in the climatic variations of interest. Data that is aggregated to large scales is generally less problematic, and different solutions come at different computational cost and may be appropriate in different contexts. Schlenker \& Roberts (2009) proposed applying "Conley" spatial standard errors (Conley, 1999) that non-parametrically estimate the variance-covariance matrix of $\beta$ by estimating $\operatorname{cov}\left(\epsilon_{i, \tau}, \epsilon_{j, \tau}\right)$ using $\xi(D(i, j)) \hat{\epsilon}_{i, \tau} \hat{\epsilon}_{j, \tau}$, where $\xi(D(i, j))$ is a kernel function that weights these terms based on $D(i, j)$, the distance between observations $i$ and $j$. Hsiang (2010) combined this approach with "Newey-West" heteroskedastic" and auto-correlation robust (HAC) standard errors (Newey \& West, 1987) to also account for temporal auto-correlation within panel units. Hsiang \& Jina (2014) demonstrated this "spatial-HAC" adjustment was correctly sized in one context by estimating

\footnotetext{
${ }^{7}$ Note that the Conley approach employed by Schlenker \& Roberts (2009) was also robust to heteroskedasticity.
} 
pseudo-exact p-values via randomizing their data in multiple dimensions and re-estimating their model many times. Fetzer (2014) expanded this approach to an instrumental variables context.

The spatial-HAC approach is computationally intensive, since distances between every pair of observations must be computed and transformed, and it does not guarantee a positive-definite estimate for the covariance matrix of residuals. Thus, it may often be reasonable to estimate approximate standard errors using simpler techniques, verifying that spatial-HAC adjustments do not alter the result substantively. For example, Dell et al. (2012) simply cluster their standard errors within panel units to account for temporal auto-correlation. Burke \& Emerick (forthcoming) cluster standard errors for county-level observations in a long-differences model by state to account for within-state spatial correlation, cross-state residual correlation in errors are assumed to be small after conditioning on state fixed effects. Hsiang et al. (2013) employ a block-boostrap in a fully non-parametric regression, blockresampling entire cross-sections of a panel data set to account for spatial autocorrelation among contemporary observations. Hsiang et al. (2011) collapse a global panel to a single time-series when examining ENSO effects, since the treatment generates spatial correlations at continental (or larger) scales.

It remains an open question what the most general and efficient approach to estimating statistical uncertainty is in most climate econometrics applications. For example, what is the optimal selection of kernel weighting functions for contemporaneous and serial observations in the spatial-HAC approach? Also, many climate data sets are derived from gridded data, which themselves might be spatially interpolated from station data or augmented with a physicsbased model-such as reanalysis products (Auffhammer et al., 2013)-and it remains unknown how these procedures influence the statistical uncertainty of resulting parameter estimates.

\subsection{Adaptation}

As discussed above, climate affects economic outcomes through belief effects and direct effects, and it is generally thought that most belief effects are adjustments that individuals make to cope with their expected distribution of direct effects. For this reason, belief effects are often described as "adaptations" to a climate, although this need not always be true (for example, beliefs about the climate could serve simply as a coordinating mechanism). In this framework, adaptations can be defined as belief effects that interact with direct effects - for example, an agent believes it will be cold sometimes at a location, causing them to purchase a coat (a belief effect) which reduces the chance they become ill after cold days (a direct effect). Multiple approaches have been used to document and quantify these adaptations.

\subsubsection{Indirect measurement via cross-section of levels}

One strategy for measuring the influence of adaptations is to estimate the effect of climate on some outcome that is influenced by adaptation using a cross-sectional research design (Eq. 8). The central benefit of this approach is that it captures all belief effects, including adaptations that interact with direct effects of the climate. For example, farm prices in Mendelsohn et al. (1994) should reflect any effects that beliefs over $\mathbf{C}$ have, including the net present value of all future revenues that result from realizations of $\mathbf{c}$, which are mediated by these beliefs and the resulting management practices. There are two weaknesses to measuring adaptations using this approach: measurement relies on the strongest form of the unit homogeneity assumption (Eq. 6) and the cross-sectional approach cannot separately disentangle belief effects that do not interact with direct effects, belief effects that do, and the integrated effect of all direct effects. However, an approach proposed by Moore \& Lobell (2014) combines this approach with time-series identification in an effort to partially isolate these effects from one another. 


\subsubsection{Explicit observation of adaptation}

Another approach to documenting adaptations is to estimate the effect of climate directly on outcomes that are known (or thought) to be adaptations to climate. For example, Hornbeck (2012) and Hidalgo et al. (2010) estimate the effect of drought on migration of agricultural households, and Kurukulasuriya \& Mendelsohn (2007) measures how climate influences the choice of crops that farmers choose to plant. This approach can be adopted in a crosssection, times-series, or long-difference framework. A benefit is that the adaptive action is known and observed directly, rather than indirectly. However, a limitation is that this approach does not recover the overall effectiveness of these adaptations, i.e. the extent to which the altered actions interact with direct effects of climate.

\subsubsection{Measurement of implicit adaptation combining time-series variation with stratification}

The one approach able to isolate the effectiveness of adaptations is to use a time-series research design (Eq. 10) for an outcome affected by adaptation, stratifying the sample - or estimating interactions - using variables that are thought to predict the extent of adaptation. For example, Auffhammer \& Aroonruengsawat (2011) estimates the effect of daily temperature on energy consumption while stratifying by long-run average temperatures, demonstrating that energy demand is higher on hot days in counties that are usually hotter on average. This result suggests that air-conditioning adoption, which is unobserved but assumed to be higher in counties that are hotter on average, increases the effect of temperature on electricity demand. Roberts \& Schlenker (2011) effectively "stratify" a panel of counties by year, implemented by interacting a response function with a nonlinear trend, to understand if innovation over time or learning reduced the heat sensitivity of maize in Indiana (Lobell et al. (2014) ask a similar question by examining how cross-sectional estimates of climatic effects on yields evolve over a sequence of years). Hsiang \& Narita (2012) derived a theory describing when such stratification works to reveal the total effectiveness of adaptations, highlighting as a benefit of this approach that it measures the overall net effectiveness of all adaptive actions that project onto the interacted proxy variables, as well as a weakness that the costs of indirectly observed adaptions are unknown. To partially address this weakness, Schlenker et al. (2013) propose an approach to measure adaptation costs in terms of the outcome variable, although it is possible that additional costs or benefits may be unobserved.

Another key challenge of this approach is that those measures used as correlates for adaptions, such as income (Hsiang \& Narita, 2012), urban status (Burgess et al., 2014), historical experience with climatic events (Hsiang \& Jina, 2014), or access to crop insurance (Annan \& Schlenker, 2015), are not exogenous and vary primarily in cross-section. This means it may be difficult to determine whether changes to the measured variable are a cause of adaptation, an effect of adaptation, or driven by an omitted variable that determines both. This drawback can be

partially solved in cases where plausibly exogenous circumstances change an influential factor, enabling a researcher to more credibly identify whether a specific factor constrains adaptation. This approach is applied by Hornbeck \& Keskin (2015) to estimate the effect of groundwater discovery on agricultural adaptation and Barreca et al. (2013) to estimate the effect of a residential air-conditioning subsidy on health-related adaptation.

\subsection{Comparisons and synthesis of results across studie}

Unlike many econometric studies, such as those that study policy changes, regressors in climate econometric studies are generally physical quantities that have similar or identical meaning at all times and at any location on the planet. Because of this, comparisons across contexts are thought to have clearer interpretations, often demonstrating replicability or highlighting important differences across samples. For example, Hsiang \& Narita (2012) and Hsiang \& Jina (2014) demonstrate notable global uniformity in the response to cyclones. In some cases, such as Guo et al. (2014) examining mortality and Hsiang et al. (2013) examining social conflict, standardization of c to a z-score based on historical variance brings parameter estimates into alignment-perhaps because populations form beliefs 
and adapt effectively to distributions of historical conditions.

In some sectors, notably agricultural impacts and climatic effects on social conflict, explicit comparisons of seemingly contradictory findings have generated substantial controversy. In the case of agriculture, much of this disagreement can be reconciled by accounting for inconsistent aggregation of data in the presence of local nonlinearities (see Section 4.1). In the case of social conflict, much of this disagreement can be reconciled by accounting for statistical uncertainty in parameter estimates (Hsiang et al., 2015; Hsiang \& Meng, 2014).

Hierarchical meta-analysis has played a role synthesizing generalizable findings and quantifying the extent of agreement in the literature (Hsiang et al., 2013) as well as to construct composite estimates for use in climate projections discussed below (Houser et al., 2015). These approaches do not assume globally uniform effects but instead model parameter estimates from the literature in a random-effects framework, where populations experience different "true" effects of the climate but may exhibit a generalizable component that is common across populations (Burke et al., 2015b; Gelman et al., 2004).

\section{ATTRIBUTION AND PROJECTION}

Two objectives of understanding the effect of climate on societies are to understand what elements of the modern world might be attributable to climatic factors and to inform projections of future outcomes under different climate scenarios. Both are cases where where parameters recovered empirically are "put to work." Note that in the following, I retain only the time index for simplicity.

\subsection{Historical attribution}

Having identified the effect of current and previous climatic conditions $\mathbf{C}$ on outcome $Y$, it is natural to ask "what counterfactual outcomes would we have observed historically under a different climate?" In our one realization of history, we observed $Y_{t}$ and $\mathbf{C}_{t}$ and estimated a response surface $\hat{f}(\mathbf{C})$ that described deviations from some benchmark outcome $Y_{0}$ associated with the benchmark climate $\mathbf{C}_{0}$. In estimation, these benchmark levels are usually nuisance parameters absorbed by various fixed effects, trends, and controls. Observed outcomes are then

$$
Y_{t}=Y_{0}+\hat{f}\left(\mathbf{C}_{t}\right)-\hat{f}\left(\mathbf{C}_{0}\right)
$$

where $Y_{0}$ can be solved for, but is not observed. Writing an analogous equation for an arbitrary counterfactual climate $\mathbf{C}_{t}+\Delta \mathbf{C}_{t}$ and an associated unknown counterfactual outcome $Y_{t}+\Delta Y_{t}$, we difference these equations to obtain

$$
\Delta \hat{Y}_{t}=\hat{f}\left(\mathbf{C}_{t}+\Delta \mathbf{C}_{t}\right)-\hat{f}\left(\mathbf{C}_{t}\right)
$$

which allows us to estimate $\Delta \hat{Y}_{t}$, the alteration of an outcome that we would expect due to a change in historical climate by $\Delta \mathbf{C}_{t}$. This approach was used by Lobell et al. (2011) to estimate the historical effect of observed warming on global crop yields, by Hsiang et al. (2011) to estimate historical influence of ENSO on global conflict, by Hsiang \& Jina (2014) to estimate historical influence of tropical cyclones on national income trajectories, and by Carleton \& Hsiang (2016) to attribute impacts based on a variety of results from the literature. Importantly, these estimates should be viewed as partial equilibrium estimates insofar as $\hat{f}($.$) captures a partial equilibrium response. Costinot$ et al. (forthcoming) and Desmet \& Rossi-Hansberg (2015) demonstrate more structured approaches that can be used to attribute historical impacts in a general-equilibrium framework.

Application of Eq. 37 must be implemented cautiously, since counterfactual outcomes are not observed and

thus cannot be verified. One indirect test of this approach, useful when $\hat{f}($.$) is identified via time-series variation,$ is to examine how closely predictions from Eq. 37 match historical cross-sectional patterns. Dell et al. (2009) run 
such a test for effects of temperature on income, arguing that adaptation and growth convergence must explain the difference. Graff Zivin et al. (2015) arrive at similar conclusions when comparing time-series estimates with longdifferences in measures of human capital. Hsiang \& Jina (2015) compares predictions based on micro-level estimates with macro-level cross sections for tropical cyclone impacts and concludes that results are largely consistent. In a remarkable higher-order test, Barreca et al. (2015) find that cross-sectional variation in the intra-annual variance in temperature, when applied to Eq. 37, is a good predictor of cross-sectional patterns of intra-annual variance in birth rates.

\subsection{Projecting future effects of climate changes}

Projecting impacts of climate changes is analogous to application of Eq. 37, except $\mathbf{C}_{t}$ is replaced with a benchmark future scenario - usually a "no change" scenario based on historical distributions of variables - and $\Delta \mathbf{C}_{t}$ is an anthropogenic alteration to the climate. Early economic analyses used simple, spatially uniform, "ballpark" estimates of $\Delta \mathbf{C}_{t}$, such as imposing a flat $+5^{\circ} \mathrm{F}$ warming and $+8 \%$ rainfall across the USA (Mendelsohn et al., 1994). In the econometrics literature, Schlenker et al. (2006) and Deschênes \& Greenstone (2007) introduced the use of spatially and temporally resolved global climate model simulations to construct $\Delta \mathbf{C}_{t}$. Lobell et al. (2008) and Burke et al. (2009) demonstrated that when applying climate model projections in Eq. 37, accounting for climate model uncertainty in $\Delta \mathbf{C}_{t}$ may be as important as accounting for statistical uncertainty in $\hat{f}($.$) See Burke et al.$ (2015a) for additional exploration of this issue.

The above approaches generally assume that $\hat{f}($.$) has a fixed structure throughout the duration of the projection$ simulation, perhaps a reasonable assumption in cases where historical changes in $\hat{f}($.$) have been limited. However,$ as demonstrated in Section 2.4 (recall Fig. 3D), the marginal effect of climate identified via weather and captured in $\hat{f}($.) may become increasingly incorrect as climatic conditions deviate from baseline conditions during a projection simulation as adaptations (or other factors, such as those described in Lobell et al. (2014)) alter $\hat{f}($.). To partially address this issue, Houser et al. (2015) demonstrated how multiple empirical estimates, capturing both crosssectional heterogeneity and trends in $\hat{f}($.$) , could be combined to construct projections where \hat{f}($.$) evolves throughout$ the projection simulation to reflect historical patterns and rates of adaptation.

\subsubsection{Top-down and bottom-up approaches}

Optimal climate policy requires understanding the full economic burden of potential climate trajectories. Empirical estimates can be used to generate projections of this total cost using either a "top-down" estimate, where the modeled outcome $Y_{t}$ is some aggregate proxy for wellbeing, such as GDP (Burke et al., 2015c; Dell et al., 2012; Deryugina \& Hsiang, 2014; Nordhaus, 2006), or constructing "bottom-up" estimates for multiple outcomes representing different sectors of the economy that are modeled and summed, sometime called the "enumerative approach" (Houser et al., 2015; Tol, 2002). In principle, both approaches can be comprehensive, so long as top-down estimates are augmented with nonmarket impacts. In practice, bottom-up estimates may be better able to account for distributional costs of climate change (Houser et al., 2015), although it is theoretically possible for them to perform equally well. When applying Eq. 37 to bottom-up projections, it is important to account for the covariance of impacts across different sectors to accurately construct the distribution of aggregate losses (Houser et al., 2015), an effect that is thought to be mostly captured in the estimated responses used for top-down projections.

\section{REMAINING CHALLENGES}

In addition to challenges described in the sections above, there are four major areas where I see methodological innovation as necessary and likely to be successful in the near future. 


\subsection{Matching effects and mechanisms}

Regardless of the research design, most estimated effects of climate are reduced form estimates that capture influences on an outcome through all possible pathways. Developing strategies and techniques that can isolate and characterize specific mechanism is critical for understanding why climatic factors matters. Testing for interactions with potential mediating factors that are plausibly exogenous (Barreca et al., 2013), exploiting natural experiments where specific pathways are shut down (Fetzer, 2014), and matching detailed patterns of climate influence on outcomes and potential mediating factors (Anttila-Hughes \& Hsiang, 2012), are all approaches that have been somewhat successful in specific contexts, although additional innovations in this area are needed as these strategies are not always available.

\subsection{Adaptation and general equilibrium}

As discussed above, adaptation to climate is thought to be economically important, but has only been characterized in a limited number of cases. Notably, the costs of adaptations are almost never measured because they are usually not observed. Moreover, most measurements are partial equilibrium responses, whereas general equilibrium responses to climate, such as factor reallocations across space or time, are a form of adaptation thought to be important but about which little is known. Further, general equilibrium changes will result in changing prices and knowing these adjustments are important for valuing quantity effects that are already understood, but only a small number of studies have begun exploring these effects (Colmer, 2016; Costinot et al., forthcoming; Dingel et al., 2015; Roberts \& Schlenker, 2013).

\subsection{Unprecedented events}

In analyses of future climate changes, valuing events that are unprecedented in recent history is a major obstacle and any empirical progress on these questions would be highly valuable. Innovative strategies that are able to measure potential costs of unprecedented physical events, such as rapid sea level rise or ocean acidification, or characterize likelihoods of unprecedented social responses to climatic changes, such as mass migrations or state failures, are needed if these impacts are to be accounted for systematically in assessment exercises.

\subsection{Integration with theory and numerical models}

Numerous theoretical models, including many used for integrated assessment policy analysis, have elements that describe climatic influence on economies (Nordhaus, 1993; Stern, 2006; Tol, 2002) but which are generally not based on empirically derived relationships. Incorporation of empirical parameter estimates into process models (Houser et al., 2015; Lobell et al., 2013) and integrated assessment models (Kopp et al., 2013; Moore \& Diaz, 2015) demonstrates promise, although much innovation is needed if these theoretical models are to perform as well as analogous models in other scientific fields. For example, it is unknown if empirical calibration improves the outof-sample forecast performance of these models or if all model parameters are even theoretically estimable using existing techniques.

\section{CONCLUSION}

Recent years have seen rapid innovation in the methods used to identify climatic influences on economies, with correspondingly rapid growth of insights that are reshaping how we understand the breadth and importance of climate-society interactions (Carleton \& Hsiang, 2016; Dell et al., 2014). Key innovations have been in research design, the measurement of climatic factors, and the formulation of econometric models. In sharp contrast to the folk 
wisdom that "climate is not weather," here I demonstrated that under fairly general conditions, weather variation, as it is used in many recent studies, exactly identifies the effect of climate - although many studies to date have not properly computed the effect of climatic changes when using these weather-derived parameters. Aggregation and synthesis of econometric findings have demonstrated a striking replicability of many recent findings across contexts, lending credibility both to the techniques that generate these results and to exercises where these results are applied to simulations of recent history or future climate changes. Many first-order partial-equilibrium results are now wellunderstood, yet major methodological innovations are still required to tackle the key challenges of identifying mechanisms, measuring adaptation costs, general equilibrium and price responses, effects of unprecedented events, and a deeper integration with theoretical models.

\section{References}

Albouy D, Graf W, Kellogg R, Wolff H. 2010. Aversion to extreme temperatures, climate change and quality of life. Working paper

Angrist JD, Pischke JS. 2008. Mostly harmless econometrics: An empiricist's companion. Princeton University Press

Annan F, Schlenker W. 2015. Federal crop insurance and the disincentive to adapt to extreme heat. American Economic Review 105:262-66

Anttila-Hughes JK, Hsiang SM. 2012. Destruction, disinvestment, and death: Economic and human losses following environmental disaster. Work. Pap., Univ. of San Francisco, San Francisco, CA

Aroonruengsawat A, Auffhammer M. 2011. Impacts of climate change on residential electricity consumption: evidence from billing data. In The Economics of climate change: Adaptations past and present. University of Chicago Press, 311-342

Auffhammer M, Aroonruengsawat A. 2011. Simulating the impacts of climate change, prices and population on california's residential electricity consumption. Climatic Change 109:191-210

Auffhammer M, Hsiang S, Schlenker W, Sobel A. 2013. Using weather data and climate model output in economic analyses of climate change. Review of Environmental Economics and Policy 7:181-198

Auffhammer M, Mansur ET. 2014. Measuring climatic impacts on energy consumption: A review of the empirical literature. Energy Economics 46:522 - 530

Auffhammer M, Ramanathan V, Vincent JR. 2006. Integrated model shows that atmospheric brown clouds and greenhouse gases have reduced rice harvests in India. Proc. Natl. Acad. Sci. 103:19668-19672

Auffhammer M, Schlenker W. 2014. Empirical studies on agricultural impacts and adaptation. Energy Economics 46:555-561

Bai Y, Kung J. 2011. Climate shocks and Sino-nomadic conflict. Rev. Econ. Stat. 93:970-981

Barreca A, Clay K, Deschenes O, Greenstone M, Shapiro JS. 2013. Adapting to climate change: The remarkable decline in the us temperature-mortality relationship over the 20th century. Tech. rep., National Bureau of Economic Research

Barreca A, Deschenes O, Guldi M. 2015. Maybe next month? temperature shocks, climate change, and dynamic adjustments in birth rates. Tech. rep., National Bureau of Economic Research

Baxter M, King RG. 1999. Measuring business cycles: approximate band-pass filters for economic time series. Review of economics and statistics 81:575-593

Baylis P. 2015. Temperature and temperament: Evidence from a billion tweets. Energy Institute Working Paper

Bertrand M, Duflo E, Mullainathan S. 2004. How much should we trust differences-in-differences estimates? Q. J. Econ. 119:249-275

Burgess R, Deschênes O, Donaldson D, Greenstone M. 2014. The unequal effects of weather and climate change: Evidence from mortality in India. Working paper

Burke M, Dykema J, Lobell DB, Miguel E, Satyanath S. 2015a. Incorporating climate uncertainty into estimates of climate change impacts. Review of Economics and Statistics 97:461-471

Burke M, Emerick K. forthcoming. Adaptation to climate change: Evidence from US agriculture. American Economic Journal: Economic Policy 
Burke M, Hsiang SM, Miguel E. 2015b. Climate and conflict. Annual Review of Economics 7:577-617

Burke M, Hsiang SM, Miguel E. 2015c. Global non-linear effect of temperature on economic production. Nature $527: 235-239$

Burke M, Miguel E, Satyanath S, Dykema J, Lobell D. 2009. Warming increases the risk of civil war in Africa. Proc. Natl. Acad. Sci. 106:20670

Camargo SJ, Hsiang SM. 2016. Tropical cyclones: From the influence of climate to their socioeconomic impacts, chap. Extreme Events: Observations, Modeling, and Economics. Wiley Online Library, 303-342

Carleton T, Hsiang S. 2016. Social and economic impacts of climate. Working paper

Colmer J. 2016. Weather, labour reallocation, and industrial production: Evidence from india. Mimeo

Conley T. 1999. GMM estimation with cross sectional dependence. Journal of Econometrics 92:1-45

Costinot A, Donaldson D, Smith C. forthcoming. Evolving comparative advantage and the impact of climate change in agricultural markets: Evidence from a 9 million-field partition of the earth. J. Polit. Econ.

Couttenier M, Soubeyran R. 2014. Drought and civil war in sub-saharan africa*. The Economic Journal 124:201-244

Dell M, Jones BF, Olken BA. 2009. Temperature and income: Reconciling new cross-sectional and panel estimates. Am. Econ. Rev. Pap. Proc. 99:198-204

Dell M, Jones BF, Olken BA. 2012. Temperature shocks and economic growth: Evidence from the last half century. American Economic Journal: Macroeconomics 4:66-95

Dell M, Jones BF, Olken BA. 2014. What do we learn from the weather? The new climate-economy literature. Journal of Economic Literature 52:740-798

Deryugina T. 2015. Disaster insurance versus social insurance in the aftermath of hurricanes. MIT Center for Energy and Environmental Policy Research working paper

Deryugina T, Hsiang SM. 2014. Does the environment still matter? Temperature and income in the United States. Working paper

Deschênes O. 2014. Temperature, human health, and adaptation: A review of the empirical literature. Energy Economics 46:606-619

Deschênes O, Greenstone M. 2007. The economic impacts of climate change: Evidence from agricultural output and random fluctuations in weather. Am. Econ. Rev. 97:354-385

Deschênes O, Greenstone M. 2011. Climate change, mortality, and adaptation: Evidence from annual fluctuations in weather in the us. Am. Econ. J. Appl. Econ. 3:152-185

Deschênes O, Moretti E. 2009. Extreme weather events, mortality and migration. Rev. Econ. Stat. 91:659-681

Desmet K, Rossi-Hansberg E. 2015. On the spatial economic impact of global warming. Journal of Urban Economics $88: 16-37$

Dingel JI, Hsiang SM, Meng KC. 2015. Global trade and risk sharing in a spatially correlated climate. Mimeo

Fankhauser S. 1995. Valuing climate change: the economics of the greenhouse. Earthscan Publications Limited

Fetzer T. 2014. Can workfare programs moderate violence? Evidence from India. Work. Pap., London School of Economics, London, UK

Fishman R. 2016. More uneven distributions overturn benefits of higher precipitation for crop yields. Environmental Research Letters 11:1-7

Gelman A, Carlin JB, Stern HS, Rubin DB. 2004. Bayesian data analysis. Chapman \& Hall/CRC

Graff Zivin J, Neidell M. 2014. Temperature and the allocation of time: Implications for climate change. J. Labor Econ. 32:1-26

Graff Zivin JS, Hsiang SM, Neidell MJ. 2015. Temperature and human capital in the short-and long-run. Tech. rep., National Bureau of Economic Research

Guiteras R, Jina A, Mobarak AM, et al. 2015. Satellites, self-reports, and submersion: Exposure to floods in bangladesh. American Economic Review 105:232-36

Guo Y, Gasparrini A, Armstrong B, Li S, Tawatsupa B, et al. 2014. Global variation in the effects of ambient temperature on mortality: a systematic evaluation. Epidemiology (Cambridge, Mass.) 25:781 
Harari M, La Ferrara E. 2013. Conflict, climate and cells: A disaggregated analysis. CEPR Discussion Paper :No. DP9277

Heal G, Park J. 2015. Goldilocks economies? temperature stress and the direct impacts of climate change. Working Paper 21119, National Bureau of Economic Research

Hidalgo F, Naidu S, Nichter S, Richardson N. 2010. Economic determinants of land invasions. Rev. Econ. Stat. 92:505-523

Hocking C, Silberstein RB, Lau WM, Stough C, Roberts W. 2001. Evaluation of cognitive performance in the heat by functional brain imaging and psychometric testing. Comparative Biochemistry and Physiology Part A: Molecular \& Integrative Physiology 128:719-734

Holland P. 1986. Statistics and causal inference. J. Am. Stat. Assoc. 81:945-960

Hornbeck R. 2012. The enduring impact of the American Dust Bowl: Short and long-run adjustments to environmental catastrophe. Am. Econ. Rev. 102:1477-1507

Hornbeck R, Keskin P. 2015. Does agriculture generate local economic spillovers? short-run and long-run evidence from the ogallala aquifer. American Economic Journal: Economic Policy 7:192-213

Houser T, Hsiang SM, Kopp R, Larsen K, Delgado M, et al. 2015. Economic Risks of Climate Change: An American Prospectus. Columbia University Press

Hsiang S, Meng K, Cane M. 2011. Civil conflicts are associated with the global climate. Nature 476:438-441

Hsiang SM. 2010. Temperatures and cyclones strongly associated with economic production in the Caribbean and Central America. Proc. Natl. Acad. Sci. 107:15367-15372

Hsiang SM, Burke M. 2014. Climate, conflict, and social stability: What does the evidence say? Climatic Change $123: 39-55$

Hsiang SM, Burke M, Miguel E. 2013. Quantifying the influence of climate on human conflict. Science 341:1235367

Hsiang SM, Burke M, Miguel E, Cane MA, Meng KC. 2015. Analysis of statistical power reconciles climate-conflict results in Africa. CEGA Working paper

Hsiang SM, Jina AS. 2014. The causal effect of environmental catastrophe on long-run economic growth: Evidence from 6,700 cyclones. Tech. rep., National Bureau of Economic Research

Hsiang SM, Jina AS. 2015. Geography, depreciation, and growth. American Economic Review 105:252-56

Hsiang SM, Meng KC. 2014. Reconciling disagreement over climate-conflict results in Africa. Proceedings of the National Academy of Sciences 111:2100-2103

Hsiang SM, Narita D. 2012. Adaptation to cyclone risk: Evidence from the global cross-section. Climate Change Economics 3:1-28

Huntington E. 1922. Civilization and climate. Yale University Press

Kopp RE, Hsiang SM, Oppenheimer M. 2013. Empirically calibrating damage functions and considering stochasticity when integrated assessment models are used as decision tools. Impacts World 2013, International Conference on Climate Change Effects.

Kousky C. 2014. Informing climate adaptation: A review of the economic costs of natural disasters. Energy Economics $46: 576-592$

Kurukulasuriya P, Mendelsohn RO. 2007. Crop selection, vol. 4307. World Bank Publications

Lobell DB, Asner GP. 2003. Climate and management contributions to recent trends in us agricultural yields. Science 299:1032-1032

Lobell DB, Burke MB, Tebaldi C, Mastrandrea MD, Falcon WP, Naylor RL. 2008. Prioritizing climate change adaptation needs for food security in 2030. Science 319:607-610

Lobell DB, Hammer GL, McLean G, Messina C, Roberts MJ, Schlenker W. 2013. The critical role of extreme heat for maize production in the united states. Nature Climate Change 3:497-501

Lobell DB, Roberts MJ, Schlenker W, Braun N, Little BB, et al. 2014. Greater sensitivity to drought accompanies maize yield increase in the us midwest. Science 344:516-519

Lobell DB, Schlenker W, Costa-Roberts J. 2011. Climate trends and global crop production since 1980. Science 333:616-620

Maccini S, Yang D. 2009. Under the weather: Health, schooling, and economic consequences of early-life rainfall. The American Economic Review 99:1006 
Mackworth NH. 1946. Effects of heat on wireless telegraphy operators hearing and recording morse messages. British Journal of Industrial Medicine 3:143-158

Mas-Colell A, Whinston MD, Green JR, et al. 1995. Microeconomic theory, vol. 1. Oxford university press New York

McCord G. forthcoming. Malaria and climate change. European Journal of Physics Special Topics

Mendelsohn R, Emanuel K, Chonabayashi S, Bakkensen L. 2012. The impact of climate change on global tropical cyclone damage. Nature Climate Change 2:205-209

Mendelsohn R, Nordhaus WD, Shaw D. 1994. The impact of global warming on agriculture: A ricardian analysis. Am. Econ. Rev. 84:753-771

Miller NL, Hayhoe K, Jin J, Auffhammer M. 2008. Climate, extreme heat, and electricity demand in california. Journal of Applied Meteorology and Climatology 47:1834-1844

Moore FC, Diaz DB. 2015. Temperature impacts on economic growth warrant stringent mitigation policy. Nature Climate Change 5:127-131

Moore FC, Lobell DB. 2014. Adaptation potential of european agriculture in response to climate change. Nature Climate Change 4:610-614

Moulton BR. 1986. Random group effects and the precision of regression estimates. Journal of econometrics 32:385397

Newey WK, West KD. 1987. A simple, positive semi-definite, heteroskedasticity and autocorrelation consistent covariance matrix. Econometrica 55:703-708

Nordhaus WD. 1993. Optimal greenhouse-gas reductions and tax policy in the" dice" model. The American Economic Review :313-317

Nordhaus WD. 2006. Geography and macroeconomics: New data and new findings. Proc. Natl. Acad. Sci. 103

Nordhaus WD. 2010. The economics of hurricanes and implications of global warming. Climate Change Economics $1: 1-20$

Nybo L, Secher NH. 2004. Cerebral perturbations provoked by prolonged exercise. Progress in Neurobiology 72:223261

Pachauri RK, Allen M, Barros V, Broome J, Cramer W, et al. 2014. Climate change 2014: Synthesis report. contribution of working groups $\mathrm{i}$, ii and iii to the fifth assessment report of the intergovernmental panel on climate change

Ranson M. 2014. Crime, weather, and climate change. J. Environ. Econ. Manag. 67:274-302

Roberts MJ, Schlenker W. 2011. The evolution of heat tolerance of corn: Implications for climate change. In The Economics of Climate Change: Adaptations Past and Present. University of Chicago Press, 225-251

Roberts MJ, Schlenker W. 2013. Identifying supply and demand elasticities of agricultural commodities: Implications for the us ethanol mandate. The American Economic Review 103:2265-2295

Sarsons H. 2015. Rainfall and conflict: A cautionary tale. Journal of Development Economics 115:62-72

Schlenker W. 2010. Crop responses to climate and weather: Cross-section and panel models. In Climate Change and Food Security: Adapting Agriculture to a Warmer World, eds. D Lobell, M Burke, chap. 6. Springer, 99-108

Schlenker W, Hanemann WM, Fisher AC. 2006. The impact of global warming on us agriculture: an econometric analysis of optimal growing conditions. Review of Economics and Statistics 88:113-125

Schlenker W, Roberts M. 2009. Nonlinear temperature effects indicate severe damages to U.S. crop yields under climate change. Proc. Natl. Acad. Sci. 106:15594-15598

Schlenker W, Roberts MJ, Lobell DB. 2013. Us maize adaptability. Nature Climate Change 3:690-691

Seppanen O, Fisk WJ, Lei Q. 2006. Room temperature and productivity in office work. Tech. rep., Lawrence Berkeley National Laboratory

Stern N. 2006. Stern review: The economics of climate change. Cambridge University Press

Tol R, Wagner S. 2010. Climate change and violent conflict in Europe over the last millennium. Clim. Chang. 99:65-79

Tol RS. 2002. Estimates of the damage costs of climate change. part 1: Benchmark estimates. Environmental and resource Economics 21:47-73

Tol RSJ. 2009. The economic effects of climate change. Journal of Economic Perspectives 23:29-51 
Urban DW, Sheffield J, Lobell DB. 2015. The impacts of future climate and carbon dioxide changes on the average and variability of us maize yields under two emission scenarios. Environmental Research Letters 10:045003

Welch JR, Vincent JR, Auffhammer M, Moya PF, Dobermann A, Dawe D. 2010. Rice yields in tropical/subtropical asia exhibit large but opposing sensitivities to minimum and maximum temperatures. Proceedings of the National Academy of Sciences 107:14562-14567

Wooldridge J. 2002. Econometric analysis of cross section and panel data. The MIT press

Yang D. 2008. Coping with disaster: The impact of hurricanes on international financial flows, 1970-2002. Advances in Economic Analysis \& Policy 8:1903-1903

Zhang DD, Brecke P, Lee HF, He YQ, Zhang J. 2007. Global climate change, war and population decline in recent human history. Proc. Natl. Acad. Sci. 104:19214-19219 